\title{
Discrete Hirota dynamics for AdS/CFT
}

\author{
Árpád Hegedüs \\ Research Institute for Particle and Nuclear Physics, \\ Hungarian Academy of Sciences, \\ H-1525 Budapest 114, P.O.B. 49, Hungary
}

\begin{abstract}
Recently a set of functional equations defining the anomalous dimensions of arbitrary local single trace operators in planar $\mathcal{N}=4$ supersymmetric Yang-Mills theory has been conjectured. These functional equations take the form of a Y-system defined on a special shaped domain. This Y-system can be equivalently reformulated as a T-system defined on a "T-shaped fat hook". The elements of the T-system satisfy discrete Hirota equations. In the present paper the discrete Hirota equations for AdS/CFT are solved by means of a chain of Bäcklund transformations and as a result TT-, TQ-, and QQ-relations are obtained for AdS/CFT.
\end{abstract}




\section{Introduction}

The AdS/CFT correspondence [1] states the equivalence of $\mathcal{N}=4$ supersymmetric Yang-Mills (SYM) theory with superstrings on $A d S_{5} \times S^{5}$. Since the correspondence is a strong-weak duality it is very difficult to test and prove it. The breakthrough in this respect was the discovery of integrability on both sides of the duality [2]-[7]. On the string theory side it means that the light-cone quantized worldsheet sigma model is an integrable quantum field theory, while on the gauge theory side integrability manifests itself in the appearance of spin chains.

To test and prove AdS/CFT one has to compute anomalous dimensions of all gauge theory operators as functions of the 't Hooft coupling $\lambda=g^{2} N_{c}$ or equivalently to find the quantized energy levels of a superstring on $A d S_{5} \times S^{5}$.

Although worldsheet quantum field theories and quantum spin chains seem to be completely different systems, their solution for large quantum numbers is encoded into the same mathematical structure: the asymptotic Bethe Ansatz [7]-[14]. Nevertheless the asymptotic Bethe Ansatz cannot be the final answer to the problem, since it fails above a certain order of perturbation theory on the gauge theory side [17]-[21]. On the gauge theory side, the reason for this breakdown is the appearance of wrapping corrections coming from contributions of Feynman graphs which encompass the whole size of the spin chain. On the string theory side these corrections can be explained by contributions coming from virtual particles traveling around the cylinder.

Such wrapping corrections in leading order can be taken into account as Lüscher corrections to the energy of the superstring sigma model [15, 16, 17, 18]. Leading order wrapping corrections in $\lambda$ have been calculated for a rich set of operators [40, 41, 42]. The desired all loop exact description of anomalous dimensions (string energies) is believed to be achieved by the Thermodynamic Bethe Ansatz (TBA) approach to the superstring sigma model [22]. As a first step the TBA equations for the groud state were derived [23, 24, 25, 26, 27], then a set of functional equations, the so-called Y-system equations were proposed to define the exact anomalous dimensions of any physical operator of planar $\mathcal{N}=4$ SYM [24]. This Y-system can be equivalently reformulated as a T-system defined on a "T-shaped fat hook" [24]. The elements of the T-system satisfy discrete Hirota equations [32]. 
In previous works [33]-39] discrete Hirota equations were considered as discrete integrable soliton equations and were solved by means of a chain of Bäcklund transformations defining the so-called undressing procedure. In case of $g l(K)$ and $g l(K \mid M)$ spin chains the T-functions of the Hirota equations correspond to fused transfer matrices of the corresponding vertex model [29, 30] and in [38, 39] it was shown that the undressing procedure defined by the successive application of Bäcklund transformations is equivalent to the nesting procedure in the nested Bethe ansatz solution of the spin chains.

In the present paper we consider discrete Hirota equations as discrete integrable soliton equations and we solve them by means of a chain of Bäcklund transformations, when they are defined on a T-shaped fat-hook of arbitrary size. As a result we get TT-, TQ-, and QQ- relations for the corresponding T-system comprising the case of AdS/CFT.

The plan of the paper is as follows: In section 2. we recall the Y-system and the associated T-system for AdS/CFT and the more general setup which we solve is addressed. In section 3. we present the chain of auto-Bäcklund transformations, and the boundary conditions for the hierarchy of Hirota equations. In section 4. and 5. we derive the TQ-, TT-, and QQ-relations for the T-system defined on a T-shaped fat-hook. In section 6. the integration of Hirota equations is discussed. In section 7. we apply our results to the case of AdS/CFT. Finally, section 8 is devoted to summary and discussion. In appendices A and B identities and relations skipped from the main text are listed.

\section{Y-, and T-systems for AdS/CFT}

In [24] a Y-system which yields the exact planar spectrum of AdS/CFT was proposed. The Y-system is a set of functional equations for the functions $Y_{a, s}(u)$ of the spectral parameter $u$ in such a way that the indices of the Y-functions are defined on a certain domain of the $(a, s)$ lattice. In case of AdS/CFT the indices take values on the domain represented in figure 1 . The Y-system functional equations take the usual universal form:

$$
\frac{Y_{a, s}(u+1) Y_{a, s}(u-1)}{Y_{a+1, s}(u) Y_{a-1, s}(u)}=\frac{\left(1+Y_{a, s+1}(u)\right)\left(1+Y_{a, s-1}(u)\right)}{\left(1+Y_{a+1, s}(u)\right)\left(1+Y_{a-1, s}(u)\right)} .
$$


The Y-system of AdS/CFT is equivalent to a T-system which is an integrable discrete dynamics on a T-shaped fat-hook (figure 1.) given by the Hirota equations [24, 25]:

$$
T_{a, s}(u+1) T_{a, s}(u-1)=T_{a+1, s}(u) T_{a-1, s}(u)+T_{a, s+1}(u) T_{a, s-1}(u),
$$

where

$$
Y_{a, s}(u)=\frac{T_{a, s+1}(u) T_{a, s-1}(u)}{T_{a+1, s}(u) T_{a-1, s}(u)}, \quad 1+Y_{a, s}(u)=\frac{T_{a, s}(u+1) T_{a, s}(u-1)}{T_{a+1, s}(u) T_{a-1, s}(u)} .
$$
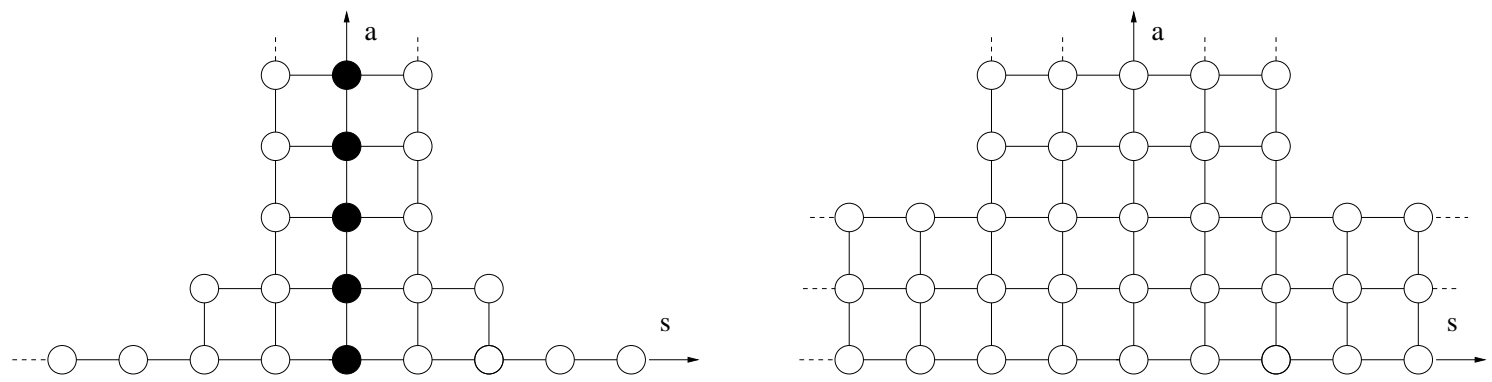

Figure 1: The domain of definition for the Y-system (on the left) and for the T-system (on the right).

Our purpose is to solve the T-system of AdS/CFT, which means that we express the infinitely many $T_{a, s}(u)$ components of the T-system with a few appropriate functions. These functions will be called the Q-functions, and they are nothing but the functions defining the boundary conditions of the hierarchy of Hirota equations generated by the Bäcklund transformations defined in the next section.

In order to be able to achieve this plan we need to consider T-systems (2) on a more general shaped domain shown in figure 2. The domain can be characterized by four integer numbers corresponding to its corner point coordinates $\left(K^{\prime},-M^{\prime}\right)$ and $(K, M)$, where the first coordinate means the " $a$-coordinate", while the second coordinate means the "s-coordinate" of the corner points on the $(a, s)$ lattice. For the sake of simplicity a T-shaped fat hook with corner point coordinates $\left(K^{\prime},-M^{\prime}\right)$ and $(K, M)$ is denoted by $\left(K^{\prime}, M^{\prime}\right) \odot(K, M)$ and will be referred as T-shaped fat hook of type $\left(K^{\prime}, M^{\prime}\right) \odot(K, M)$. The T-functions corresponding to such a domain will be demoted by $T_{K, M}^{K^{\prime}, M^{\prime}}(a, s, u)$. The AdS/CFT case corresponds to the special $K^{\prime}=M^{\prime}=K=M=2$ choice for the domain. An important property of the 


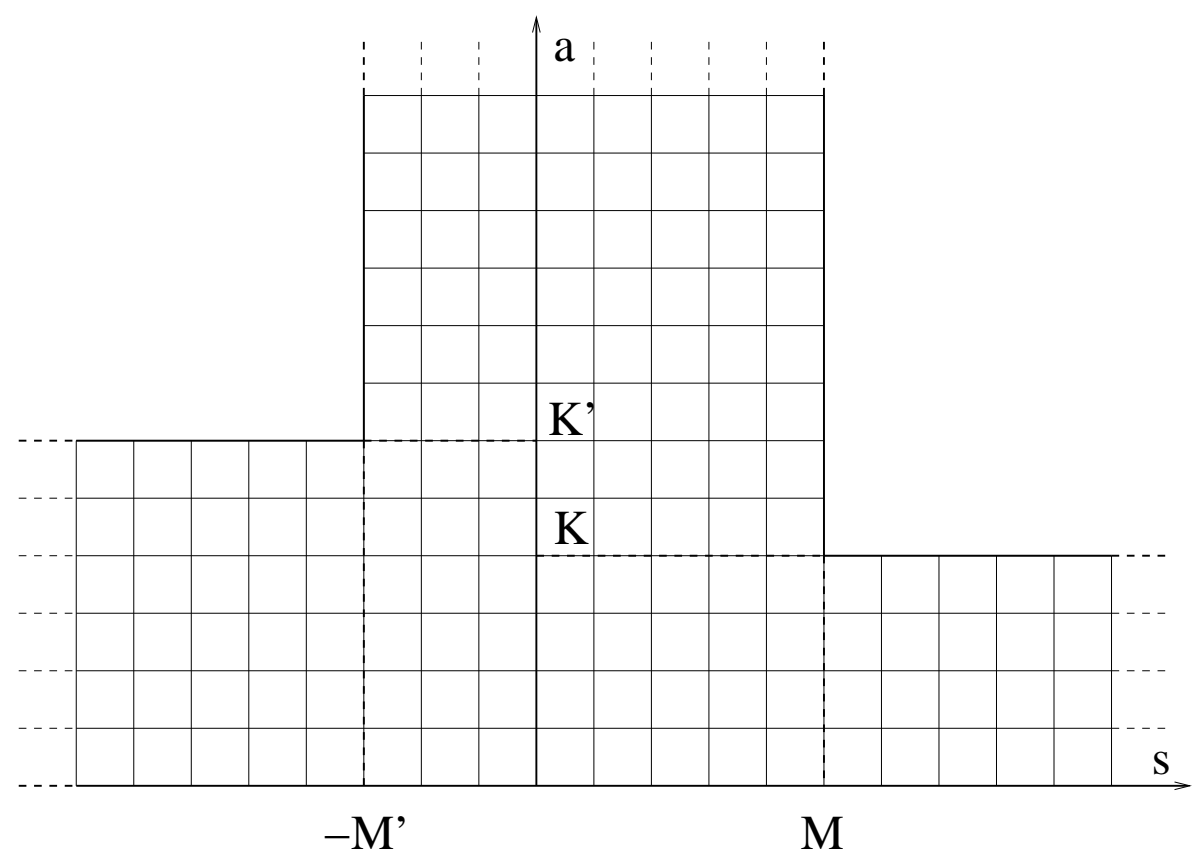

Figure 2: General T-shaped fat hook .

Hirota equations is their invariance with respect to the gauge transformations:

$$
T_{a, s}(u) \rightarrow g_{1}(u+a+s) g_{2}(u+a-s) g_{3}(u-a+s) g_{4}(u-a-s) T_{a, s}(u) .
$$

Both Y- and T-systems are sets of functional equations which have to be supplemented with certain boundary conditions on the $(a, s)$ lattice. Our choice for the boundary conditions of a T-shaped fat hook of type $\left(K^{\prime}, M^{\prime}\right) \odot(K, M)$ will be specified in the next section.

\section{Hierarchy of Hirota equations}

This section is devoted to the discussion of the boundary conditions and autoBäcklund transformations of the Hirota equations defined on a T-shaped fat-hook represented in figure 2 . We begin with the boundary conditions. 


\subsection{Boundary conditions for T-shaped fat-hook}

We extend the Hirota equations (2) to be valid on the whole $(a, s)$ lattice, in such a way that the $T_{a, s}(u)$ functions are zero if their indices take values outside of the T-shaped fat hook of type $\left(K^{\prime}, M^{\prime}\right) \odot(K, M)$ :

$$
T(a, s, u)=0, \quad \text { if: (i) } a<0 \text {, or (ii) } a>K, s>M \text { or (iii) } a>K^{\prime}, s<-M^{\prime} \text {, }
$$

where we introduced the notation $T_{a, s}(u)=T(a, s, u)$. The boundary values of $T(a, s, u)$ are rather special, because taking the Hirota equations (2) at the boundaries they reduce to discrete d'Alambert equations. For example, taking (2) at $a=0$, the equations reduce to:

$$
T(0, s, u+1) T(0, s, u-1)=T(0, s-1, u) T(0, s+1, u) .
$$

The solution of these equations can be expressed as a product of two arbitrary functions, one is the function of $u-s$ only and the other depends only on $u+s$ :

$$
T(0, s, u)=f_{-}(u-s) f_{+}(u+s) .
$$

This analysis can be done to all boundary lines of the T-shaped fat-hook and finally one gets that the boundary values of $T(a, s, u)$ along the horizontal (vertical) boundaries are products of two functions, one depending only on $u-s,(u-a)$ and the other depending only on $u+s,(u+a)$. There is an additional constraint that connects the horizontal and vertical boundary values of $T(a, s, u)$, namely because the corner points $(K, M)$ or $\left(K^{\prime},-M^{\prime}\right)$ are part of both the vertical and horizontal boundary lines, the horizontal and vertical boundary values for $T(a, s, u)$ must be equal at these points. It turns out that the most general boundary conditions satisfying the previous conditions can be brought by an appropriate gauge transformation (4) into the following form:

$$
\begin{array}{rlrl}
T(0, s, u) & =Q^{K^{\prime}, M^{\prime}}(u+s) \tilde{Q}_{K, M}(u-s) & -\infty<s<\infty & \\
T\left(K^{\prime}, s, u\right) & =\mathcal{Q}_{K, M}^{K^{\prime}}\left(u-s+K^{\prime}\right) Q^{0, M^{\prime}}\left(u+s-K^{\prime}\right) & s \leq-M^{\prime} \\
T\left(a,-M^{\prime}, u\right) & =(-1)^{M^{\prime}\left(a-K^{\prime}\right)} \mathcal{Q}_{K, M}^{K^{\prime}}\left(u+a+M^{\prime}\right) Q^{0, M^{\prime}}\left(u-a-M^{\prime}\right) & a \geq K^{\prime} \\
T(K, s, u) & =\tilde{\mathcal{Q}}_{K}^{K^{\prime}, M^{\prime}}(u+s+K) \tilde{Q}_{0, M}(u-s-K) & s \geq M \\
T(a, M, u) & =(-1)^{M(a-K)} \tilde{\mathcal{Q}}_{K}^{K^{\prime}, M^{\prime}}(u+a+M) \tilde{Q}_{0, M}(u-a-M) & a \geq K .
\end{array}
$$


The functions appearing on the right hand side of (7) characterize the boundary conditions for $T(a, s, u)$. In the present paper our purpose is to solve the Hirota equations (2) when boundary conditions (7) are imposed.

Our strategy of solving Hirota equations agrees with the method worked out in [38]. Namely, we consider the Hirota equations as discrete integrable soliton equations, and by giving their appropriate auto-Bäcklund transformations we can construct a hierarchy of Hirota equations, in such a way that two neighboring levels of the hierarchy are connected by a Bäcklund transformation. The hierarchy of Hirota equations consists of Hirota equations defined on T-shaped fat hooks with different corner point coordinates. Two members of the hierarchy are called neighboring, when only one of the four corner point coordinates of their T-shaped fat hooks differ by 1 . (i.e. one of the parameters $K^{\prime}, M^{\prime}, K, M$ differ by 1 ). The existence of such a hierarchy follows from the classical integrability of the Hirota equations. With the help of the Bäcklund transformations one can decrease $K, M$ or $K^{\prime}, M^{\prime}$ by 1 , "undressing" step by step the original $\left(K^{\prime}, M^{\prime}\right) \odot(K, M)$ problem to the trivial $(0,0) \odot(0,0)$ or $(0,-m) \odot(0, m)$ problem. Then taking the "inverse" of the undressing procedure the solution for the Hirota equations can be built up from the trivial $(0,0) \odot(0,0)$ problem. This procedure will be explained in section 6.

The "undressing" procedure was shown to be equivalent to algebraic Bethe ansatz in case of $g l(K)$ [37, 38] and $g l(K \mid M)$ [39] spin chains. In the $g l(K)$ case the domain of non-zero $T(a, s, u)$ is a semi-infinite strip of height $\mathrm{K}$ [38], while in case of $g l(K \mid M)$ super algebras this domain is a fat-hook with corner point coordinates $(K, M)$ [39].

In the next subsection we define the auto-Bäcklund transformations of the Hirota equations which provide us with a hierarchy of Hirota equations.

\subsection{Bäcklund transformations}

In this subsection we define 4 auto-Bäcklund transformations of the Hirota equations each corresponding to shifting one of the coordinates $K, M, K^{\prime}, M^{\prime}$ by 1 unit. We use the terminology of left and right Bäcklund transformations in the following sense: if a Bäcklund transformation decreases the value of $K$ or $M$ by 1 we call it right Bäcklund transformation, and if it decreases the value of $K^{\prime}$ or $M^{\prime}$ we call it left 
Bäcklund transformation. Right Bäcklund transformations change the corner point coordinates of the T-shaped fat-hook on the right hand side and leave the left hand side intact, while the left Bäcklund transformations change the T-shaped fat-hook on the left hand side and leave the right hand side intact. The right Bäcklund transformations take exactly the same form as those defined for the $g l(K \mid M)$ super spin chains [39]. Let us call the Bäcklund transformed functions $F(a, s, u)$, then the defining equations of the right Bäcklund transformations read as [39]:

$$
\begin{array}{r}
T(a+1, s, u) F(a, s, u+1)-T(a, s, u+1) F(a+1, s, u)= \\
T(a+1, s-1, u+1) F(a, s+1, u), \\
T(a, s+1, u+1) F(a, s, u)-T(a, s, u) F(a, s+1, u+1)= \\
T(a+1, s, u+1) F(a-1, s+1, u) .
\end{array}
$$

or equivalently after some simple manipulations described in [39] it can be written as a single matrix equation

$$
\mathbb{T} \mathbf{F}=0
$$

where the matrix $\mathbb{T}$ is given by:

$$
\mathbb{T}=\left(\begin{array}{cccc}
0 & T(a, s, u-1) & -T(a, s+1, u) & T(a+1, s, u) \\
-T(a, s, u-1) & 0 & T(a-1, s, u) & T(a, s-1, u) \\
T(a, s+1, u) & -T(a-1, s, u) & 0 & -T(a, s, u+1) \\
-T(a+1, s, u) & -T(a, s-1, u) & T(a, s, u+1) & 0
\end{array}\right)
$$

and the vector $\mathbf{F}$ reads as:

$$
\mathbf{F}=\left(\begin{array}{c}
F(a-1, s, u) \\
F(a, s+1, u) \\
F(a, s, u-1) \\
F(a-1, s+1, u-1)
\end{array}\right)
$$

It can be seen that the right Bäcklund transformations are homogeneous linear equations for $F(a, s, u)$ which have nontrivial solutions only if the determinant of the matrix $\mathbb{T}$ vanishes. In fact this happens because $T(a, s, u)$ satisfies the Hirota equations, and it turns out that the rank of the matrix $\mathbb{T}$ is 2 , so there are 2 linearly independent solutions of the linear problem (9)). 
There is a duality between $T(a, s, u)$ and $F(a, s, u)$ [39], namely one can interchange the roles of $T$ and $F$ and consider (8) as an over-determined system of linear equations for $T$ with coefficients $F$. Their compatibility equation says that $F(a, s, u)$ satisfies the Hirota equations (2) as well. However it can be shown that $F$ can not be defined on the same T-shaped fat-hook as $T$. Careful analysis of the boundary conditions for $F$ compatible with (8) says that $F$ must be defined either on a T-shaped fat-hook of type $\left(K^{\prime}, M^{\prime}\right) \odot(K-1, M)$ or on a T-shaped fat-hook of type $\left(K^{\prime}, M^{\prime}\right) \odot(K, M+1)$. These two possible choices for the domain of definition correspond to the 2 linearly independent solutions of (9). We want such right Bäcklund transformations which decrease the value of $K$ or $M$ by 1 unit. The first ( $K$ decreasing) right Bäcklund transformation (BT1) is defined by equations (8), while the second ( $M$ decreasing) right transformation can be obtained from (8) by interchanging the role of $T$ and $F[39]$. We denote it (BT2) for short, and it reads:

$$
\begin{array}{r}
F^{*}(a+1, s, u) T(a, s, u+1)-F^{*}(a, s, u+1) T(a+1, s, u)= \\
F^{*}(a+1, s-1, u+1) T(a, s+1, u), \\
F^{*}(a, s+1, u+1) T(a, s, u)-F^{*}(a, s, u) T(a, s+1, u+1)= \\
F^{*}(a+1, s, u+1) T(a-1, s+1, u) .
\end{array}
$$

The function $F^{*}(a, s, u)$ is defined on a T-shaped fat-hook of type $\left(K^{\prime}, M^{\prime}\right) \odot(K, M-$ $1)$. With the help of the (BT1) and (BT2) Bäcklund transformations one can decrease the size of the T-shaped fat-hook on its right hand side by 1 unit in either vertical or horizontal direction.

In order that the T-shaped fat-hook can be reduced to a trivial domain one needs another set of Bäcklund transformations which decrease the size of the Tshaped fat-hook on its left hand side. These left Bäcklund transformations can be easily created from (8) after performing a reflection transformation in the $s$ variable. Denoting the left Bäcklund transformed function $\tilde{F}(a, s, u)$ they read as:

$$
\begin{array}{r}
T(a+1, s, u) \tilde{F}(a, s, u+1)-T(a, s, u+1) \tilde{F}(a+1, s, u)= \\
T(a+1, s+1, u+1) \tilde{F}(a, s-1, u), \\
T(a, s-1, u+1) \tilde{F}(a, s, u)-T(a, s, u) \tilde{F}(a, s-1, u+1)= \\
T(a+1, s, u+1) \tilde{F}(a-1, s-1, u) .
\end{array}
$$


or equivalently it can be written as a single matrix equation:

$$
\tilde{\mathbb{T}} \tilde{\mathbf{F}}=0
$$

where the matrix $\tilde{\mathbb{T}}$ is given by:

$$
\tilde{\mathbb{T}}=\left(\begin{array}{cccc}
0 & T(a, s, u-1) & -T(a, s-1, u) & T(a+1, s, u) \\
-T(a, s, u-1) & 0 & T(a-1, s, u) & T(a, s+1, u) \\
T(a, s-1, u) & -T(a-1, s, u) & 0 & -T(a, s, u+1) \\
-T(a+1, s, u) & -T(a, s+1, u) & T(a, s, u+1) & 0
\end{array}\right)
$$

and the vector $\tilde{\mathbf{F}}$ reads as:

$$
\tilde{\mathbf{F}}=\left(\begin{array}{c}
\tilde{F}(a-1, s, u) \\
\tilde{F}(a, s-1, u) \\
\tilde{F}(a, s, u-1) \\
\tilde{F}(a-1, s-1, u-1)
\end{array}\right)
$$

Following similar train of thoughts as in case of the right Bäcklund transformations it can be shown that (14) has also two linearly independent solutions which satisfy the Hirota equations. They correspond to T-shaped fat hooks of type $\left(K^{\prime}-1, M^{\prime}\right) \odot$ $(K, M)$ or $\left(K^{\prime}, M^{\prime}+1\right) \odot(K, M)$. Then the first $\left(K^{\prime}\right.$ decreasing) left Bäcklund transformation $(\overline{B T 1})$ is defined by equations (13), while the second ( $M^{\prime}$ decreasing) transformation can be obtained from (13) by interchanging the role of $T$ and $\tilde{F}$. We call this second left Bäcklund transformation $(\overline{B T 2})$, and it reads:

$$
\begin{array}{r}
\tilde{F}^{*}(a+1, s, u) T(a, s, u+1)-\tilde{F}^{*}(a, s, u+1) T(a+1, s, u)= \\
\tilde{F}^{*}(a+1, s+1, u+1) T(a, s-1, u), \\
\tilde{F}^{*}(a, s-1, u+1) T(a, s, u)-\tilde{F}^{*}(a, s, u) T(a, s-1, u+1)= \\
\tilde{F}^{*}(a+1, s, u+1) T(a-1, s-1, u) .
\end{array}
$$

The function $F^{*}(a, s, u)$ is defined on a T-shaped fat-hook of type $\left(K^{\prime}, M^{\prime}-1\right) \odot$ $(K, M)$. With the help of the $(\overline{B T 1})$ and $(\overline{B T 2})$ Bäcklund transformations one can decrease the size of the T-shaped fat-hook on its left hand side by 1 unit in either vertical or horizontal direction. The actions of (BT1), (BT2), $(\overline{B T 1})$ and $(\overline{B T 2})$ Bäcklund transformations are depicted in figure 3 . 


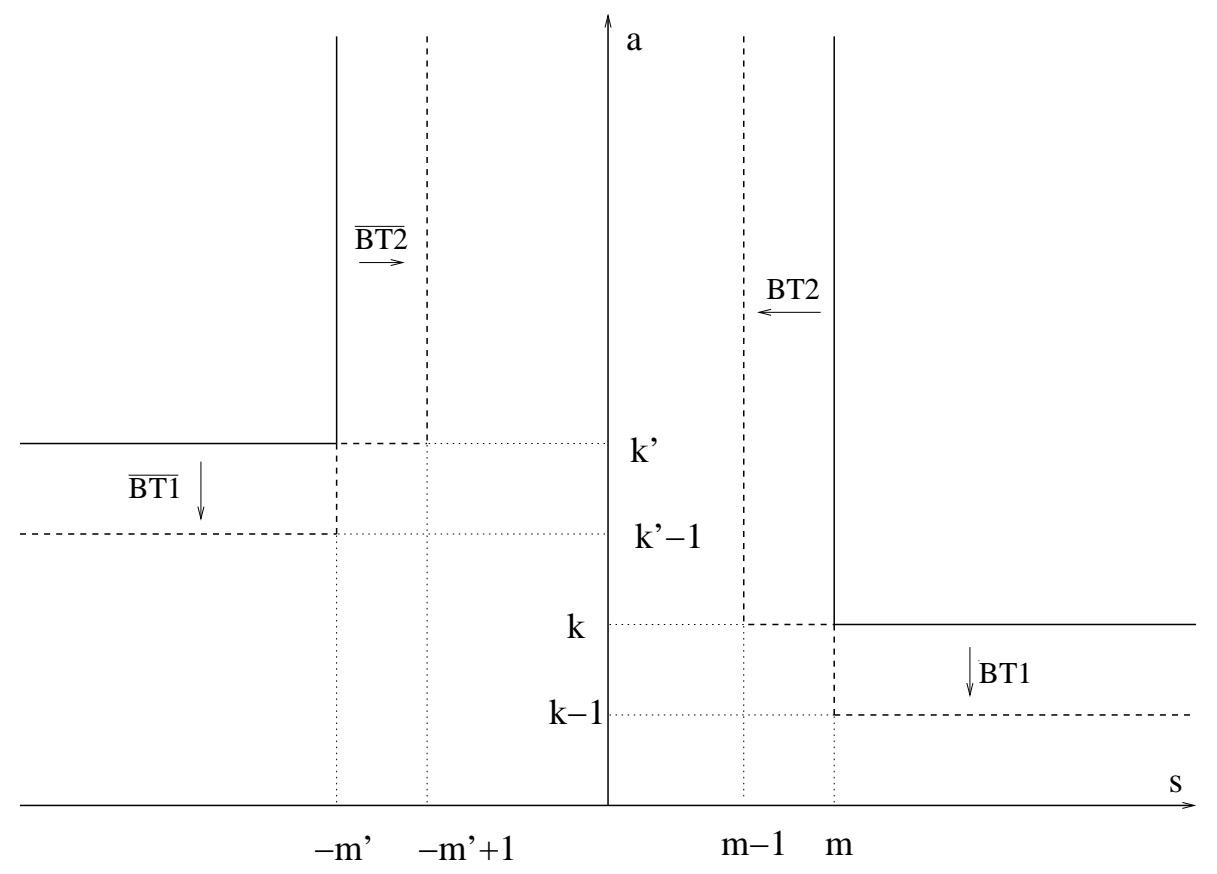

Figure 3: Schematic representation of the actions of (BT1), (BT2), $(\overline{B T 1})$ and $(\overline{B T 1})$ Bäcklund transformations.

\subsection{Boundary conditions for the hierarchy of Hirota equa- tions}

First of all we introduce the notation $T_{k, m}^{k^{\prime}, m^{\prime}}(a, s, u)$ for T-functions, which solve the Hirota equations defined on a T-shaped fat-hook of type $\left(k^{\prime}, m^{\prime}\right) \odot(k, m)$. With the help of this new notation the matrix form of right Bäcklund transformations (8) - (12) can be written as:

BT1 case: $\quad \mathbb{T}_{k, m}^{k^{\prime}, m^{\prime}} \mathbf{T}_{k-1, m}^{k^{\prime}, m^{\prime}}=0, \quad$ BT2 case: $\quad \mathbb{T}_{k, m}^{k^{\prime}, m^{\prime}} \mathbf{T}_{k, m+1}^{k^{\prime}, m^{\prime}}=0$,

where the matrix $\mathbb{T}_{k, m}^{k^{\prime}, m^{\prime}}$ is given by:

$\mathbb{T}_{k, m}^{k^{\prime}, m^{\prime}}=\left(\begin{array}{cccc}0 & T_{k, m}^{k^{\prime}, m^{\prime}}(a, s, u-1) & -T_{k, m}^{k^{\prime}, m^{\prime}}(a, s+1, u) & T_{k, m}^{k^{\prime}, m^{\prime}}(a+1, s, u) \\ -T_{k, m}^{k^{\prime}, m^{\prime}}(a, s, u-1) & 0 & T_{k, m}^{k^{\prime}, m^{\prime}}(a-1, s, u) & T_{k, m}^{k^{\prime}, m^{\prime}}(a, s-1, u) \\ T_{k, m^{\prime}}^{k^{\prime}, m^{\prime}}(a, s+1, u) & -T_{k, m}^{k^{\prime}, m^{\prime}}(a-1, s, u) & 0 & -T_{k, m}^{k^{\prime}, m^{\prime}}(a, s, u+1) \\ -T_{k, m}^{k^{\prime}, m^{\prime}}(a+1, s, u) & -T_{k, m}^{k^{\prime}, m^{\prime}}(a, s-1, u) & T_{k, m}^{k^{\prime}, m^{\prime}}(a, s, u+1) & 0\end{array}\right)$ 


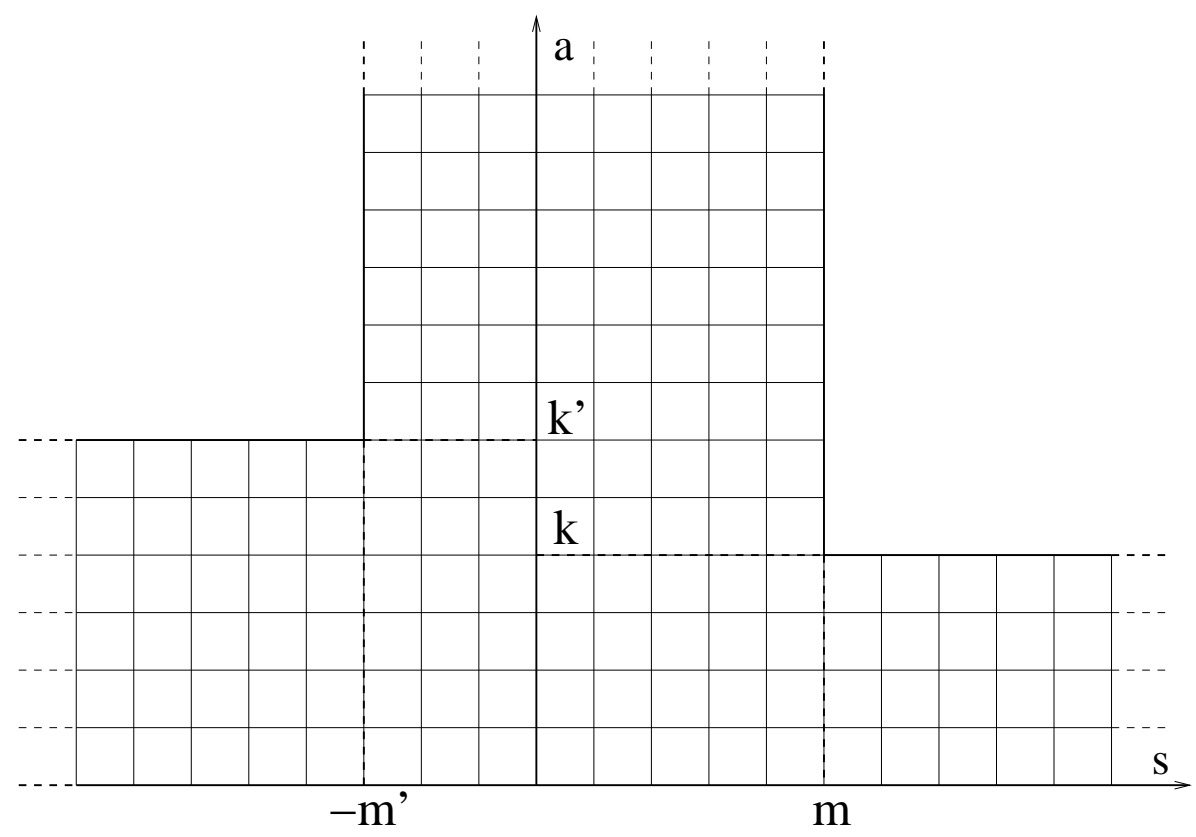

Figure 4: Domain of definition for T-functions $T_{k, m}^{k^{\prime}, m^{\prime}}(a, s, u)$.

and the vector $\mathbf{T}_{k, m}^{k^{\prime}, m^{\prime}}$ reads as:

$$
\mathbf{T}_{k, m}^{k^{\prime}, m^{\prime}}=\left(\begin{array}{c}
T_{k, m}^{k^{\prime}, m^{\prime}}(a-1, s, u) \\
T_{k, m}^{k^{\prime}, m^{\prime}}(a, s+1, u) \\
T_{k, m}^{k^{\prime}, m^{\prime}}(a, s, u-1) \\
T_{k, m}^{k^{\prime}, m^{\prime}}(a-1, s+1, u-1)
\end{array}\right)
$$

The matrix form of left Bäcklund transformations (13)-(17) can be written as:

$$
\overline{B T 1} \quad \text { case: } \quad \tilde{\mathbb{T}}_{k, m}^{k^{\prime}, m^{\prime}} \tilde{\mathbf{T}}_{k, m}^{k^{\prime}-1, m^{\prime}}=0, \quad \overline{B T 2} \quad \text { case: } \quad \tilde{\mathbb{T}}_{k, m}^{k^{\prime}, m^{\prime}} \tilde{\mathbf{T}}_{k, m}^{k^{\prime}, m^{\prime}+1}=0,
$$

where the matrix $\tilde{\mathbb{T}}_{k, m}^{k^{\prime}, m^{\prime}}$ is given by:

$$
\tilde{\mathbb{T}}_{k, m}^{k^{\prime}, m^{\prime}}=\left(\begin{array}{cccc}
0 & T_{k, m}^{k^{\prime}, m^{\prime}}(a, s, u-1) & -T_{k, m}^{k^{\prime}, m^{\prime}}(a, s-1, u) & T_{k, m}^{k^{\prime}, m^{\prime}}(a+1, s, u) \\
-T_{k, m}^{k^{\prime}, m^{\prime}}(a, s, u-1) & 0 & T_{k, m}^{k^{\prime}, m^{\prime}}(a-1, s, u) & T_{k, m}^{k^{\prime}, m^{\prime}}(a, s+1, u) \\
T_{k, m}^{k^{\prime}, m^{\prime}}(a, s-1, u) & -T_{k, m}^{k^{\prime}, m^{\prime}}(a-1, s, u) & 0 & -T_{k, m}^{k^{\prime}, m^{\prime}}(a, s, u+1) \\
-T_{k, m}^{k^{\prime}, m^{\prime}}(a+1, s, u) & -T_{k, m}^{k^{\prime}, m^{\prime}}(a, s+1, u) & T_{k, m}^{k^{\prime}, m^{\prime}}(a, s, u+1) & 0
\end{array}\right),
$$


and the vector $\tilde{\mathbf{T}}_{k, m}^{k^{\prime}, m^{\prime}}$ reads as:

$$
\tilde{\mathbf{T}}_{k, m}^{k^{\prime}, m^{\prime}}=\left(\begin{array}{c}
T_{k, m}^{k^{\prime}, m^{\prime}}(a-1, s, u) \\
T_{k, m}^{k^{\prime}, m^{\prime}}(a, s-1, u) \\
T_{k, m}^{k^{\prime}, m^{\prime}}(a, s, u-1) \\
T_{k, m}^{k^{\prime}, m^{\prime}}(a-1, s-1, u-1)
\end{array}\right) .
$$

The (18) and (21) Bäcklund transformations relate the boundary conditions of $T_{k, m}^{k^{\prime}, m^{\prime}}(a, s, u)$ at different values of their indices. If one starts the undressing procedure from $T_{K, M}^{K^{\prime}, M^{\prime}}(a, s, u)$ with boundary conditions given by (7), then all the $T_{k, m}^{k^{\prime}, m^{\prime}}(a, s, u)$ functions of the hierarchy of Hirota equations have structurally the same type of boundary conditions:

$$
\begin{array}{rlr}
T_{k, m}^{k^{\prime}, m^{\prime}}(0, s, u) & =Q^{k^{\prime}, m^{\prime}}(u+s) \tilde{Q}_{k, m}(u-s) \quad-\infty<s<\infty & \\
T_{k, m}^{k^{\prime}, m^{\prime}}\left(k^{\prime}, s, u\right) & =\mathcal{Q}_{k, m}^{k^{\prime}}\left(u-s+k^{\prime}\right) Q^{0, m^{\prime}}\left(u+s-k^{\prime}\right) & s \leq-m^{\prime} \\
T_{k, m}^{k^{\prime}, m^{\prime}}\left(a,-m^{\prime}, u\right) & =(-1)^{m^{\prime}\left(a-k^{\prime}\right)} \mathcal{Q}_{k, m}^{k^{\prime}}\left(u+a+m^{\prime}\right) Q^{0, m^{\prime}}\left(u-a-m^{\prime}\right) & a \geq k^{\prime} \\
T_{k, m}^{k^{\prime}, m^{\prime}}(k, s, u) & =\tilde{\mathcal{Q}}_{k}^{k^{\prime}, m^{\prime}}(u+s+k) \tilde{Q}_{0, m}(u-s-k) & s \geq m \\
T_{k, m}^{k^{\prime}, m^{\prime}}(a, m, u) & =(-1)^{m(a-k)} \tilde{\mathcal{Q}}_{k}^{k^{\prime}, m^{\prime}}(u+a+m) \tilde{Q}_{0, m}(u-a-m) & a \geq k .
\end{array}
$$

The dependence of the boundary functions on their indices is dictated by the fact that one gets all the $T_{k, m}^{k^{\prime}, m^{\prime}}(a, s, u)$ functions from $T_{K, M}^{K^{\prime}, M^{\prime}}(a, s, u)$ by successive application of Bäcklund transformations (18) and (21).

At the present stage of the presentation the boundary functions on the right hand side of (24) seem to be arbitrary functions of $u$. But this is not the case. There are certain relations among them.

The first class of relations comes from such degenerate cases, when certain parts of the boundary lines of the T-shaped fat hook coincide. For example, for the special choice of $m=-m^{\prime}$, there is a common part of the vertical left and right boundary lines of the T-shaped fat-hook (see figure 5.). This degeneration connects the boundary functions on the left and right hand sides of the T-shaped fat hook. Relations coming from such coincidences of boundary lines are listed in appendix A.

The second class of relations among boundary functions of (24) are given by the so-called QQ-relations, which will be the subject of section 5 . 


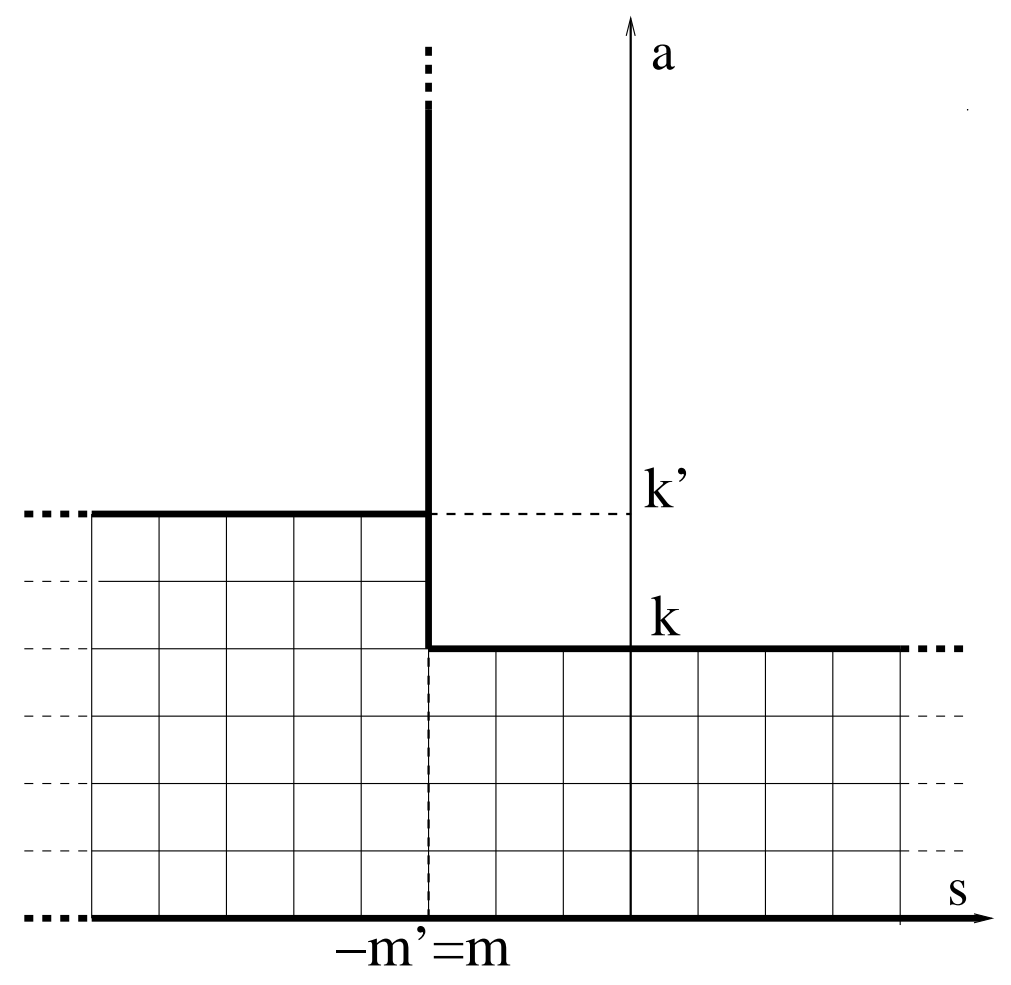

Figure 5: Degenerate domain corresponding to the choice $m=-m^{\prime}$.

\section{TQ-relations}

This short section summarizes the TQ-relations for the Hirota equations defined on the T-shaped fat-hook of type $\left(K^{\prime}, M^{\prime}\right) \odot(K, M)$. In our case generalized Baxter equations or TQ-relations are bilinear equations between the functions $T_{k, m}^{k^{\prime}, m^{\prime}}(a, s, u)$ and $Q^{k^{\prime}, m^{\prime}}(u)$ or $\tilde{Q}_{k, m}(u)$, because these bilinear relations can be considered as generalizations of the TQ-relations of the simplest and well known case of the $S U(2)$ spin chain [44]. As it was shown in [37, 38] the Q-functions of the spin chains can be identified with the functions characterizing the boundary conditions of the domain of definition of the T-functions. The set of functions one can identify as the $Q$-functions of the problem are the $Q^{k^{\prime}, m^{\prime}}(u)$ and $\tilde{Q}_{k, m}(u)$ functions. In this case the TQ-relations can be obtained by taking the (18) (BT1), (BT2), and (21), ( $\overline{B T 1}$ ), $(\overline{B T 2})$ Bäcklund transformations along the boundary line $(0, s)$. TQ-relations com- 
ing from (BT1) and (BT2) Bäcklund transformations read as follows:

$$
\begin{array}{r}
T_{k, m}^{k^{\prime}, m^{\prime}}(1, s, u) \tilde{Q}_{k-1, m}(u-s+1)-T_{k-1, m}^{k^{\prime}, m^{\prime}}(1, s, u) \tilde{Q}_{k, m}(u-s+1)= \\
T_{k, m}^{k^{\prime}, m^{\prime}}(1, s-1, u+1) \tilde{Q}_{k-1, m}(u-s-1) \\
T_{k, m-1}^{k^{\prime}, m^{\prime}}(1, s, u) \tilde{Q}_{k, m}(u-s+1)-T_{k, m}^{k^{\prime}, m^{\prime}}(1, s, u) \tilde{Q}_{k, m-1}(u-s+1)= \\
T_{k, m-1}^{k^{\prime}, m^{\prime}}(1, s-1, u+1) \tilde{Q}_{k, m}(u-s-1),
\end{array}
$$

while TQ-relations coming from $(\overline{B T 1}),(\overline{B T 2})$ Bäcklund transformations are as follows:

$$
\begin{array}{r}
T_{k, m}^{k^{\prime}, m^{\prime}}(1, s, u) Q^{k^{\prime}-1, m^{\prime}}(u+s+1)-T_{k, m}^{k^{\prime}-1, m^{\prime}}(1, s, u) Q^{k^{\prime}, m^{\prime}}(u+s+1)= \\
T_{k, m}^{k^{\prime}, m^{\prime}}(1, s+1, u+1) Q^{k^{\prime}-1, m^{\prime}}(u+s-1) \\
T_{k, m}^{k^{\prime}, m^{\prime}-1}(1, s, u) Q^{k^{\prime}, m^{\prime}}(u+s+1)-T_{k, m}^{k^{\prime}, m^{\prime}}(1, s, u) Q^{k^{\prime}, m^{\prime}-1}(u+s+1)= \\
T_{k, m}^{k^{\prime}, m^{\prime}-1}(1, s+1, u+1) Q^{k^{\prime}, m^{\prime}}(u+s-1) .
\end{array}
$$

In references [38] and [39] these TQ-relations were used to construct an operator generating series for the T-functions. The generating series was factorized into an ordered product of first order difference operators with coefficients being ratios of the $Q$-functions. Here we skip the construction of such generating series and we will discuss the problem of expressing the T-functions in terms of the $Q^{k^{\prime}, m^{\prime}}(u)$ and $\tilde{Q}_{k, m}(u)$ functions later in section 6 .

Closing this section we have to mention that the (18) and (21) Bäcklund transformations written along the right and left vertical boundary lines imply additional TQ type bilinear relations between the T-functions and the boundary characterizing functions. They are listed in Appendix B.

\section{TT- and QQ-relations}

In this section we derive bilinear equations for the functions $T_{k, m}^{k^{\prime}, m^{\prime}}(a, s, u)$ in which either both indices $k, m$ or both indices $k^{\prime}, m^{\prime}$ undergo shifts by \pm 1 . These equations are also of Hirota type and we call them TT-relations. The special cases (43) and (44) are particularly important. They provide bilinear relations for the $Q$ and $\tilde{Q}$ functions which we call QQ-relations for traditional reasons. 
All these bilinear TT-relations follow from the (BT1), (BT2) and $(\overline{B T 1}),(\overline{B T 2})$ Bäcklund transformations with shifts in $k$ and $m$ or in $k^{\prime}$ and $m^{\prime}$.Following the lines of [39] it can be shown that the first rows of (18) can be rewritten as

$$
\begin{aligned}
& \Psi_{k-1, m}^{k^{\prime}, m^{\prime}}(a, s, u)=\hat{\mathcal{A}}_{k, m}^{k^{\prime}, m^{\prime}}(a, s, u) \Psi_{k, m}^{k^{\prime}, m^{\prime}}(a, s, u), \\
& \Psi_{k, m+1}^{k^{\prime}, m^{\prime}}(a, s, u)=\hat{\mathcal{B}}_{k, m}^{k^{\prime}, m^{\prime}}(a, s, u) \Psi_{k, m}^{k^{\prime}, m^{\prime}}(a, s, u),
\end{aligned}
$$

where

$$
\Psi_{k, m}^{k^{\prime}, m^{\prime}}(a, s, u)=\frac{T_{k, m}^{k^{\prime}, m^{\prime}}(a+1, s, u)}{T_{k, m}^{k^{\prime}, m^{\prime}}(a, s+1, u)},
$$

and $\hat{\mathcal{A}}_{k, m}^{k^{\prime}, m^{\prime}}(a, s, u)$ and $\hat{\mathcal{B}}_{k, m}^{k^{\prime}, m^{\prime}}(a, s, u)$ are operators acting on the functions $\Psi_{k, m}^{k^{\prime}, m^{\prime}}(a, s, u)$

$$
\begin{aligned}
& \hat{\mathcal{A}}_{k, m}^{k^{\prime}, m^{\prime}}(a, s, u)=\frac{T_{k-1, m}^{k^{\prime}, m^{\prime}}(a, s, u+1) T_{k, m}^{k^{\prime}, m^{\prime}}(a, s+1, u)}{T_{k-1, m}^{k^{\prime}, m^{\prime}}(a, s+1, u) T_{k, m}^{k^{\prime}, m^{\prime}}(a, s, u+1)}-e^{\partial_{u}-\partial_{s}}, \\
& \hat{\mathcal{B}}_{k, m}^{k^{\prime}, m^{\prime}}(a, s, u)=\frac{T_{k, m+1}^{k^{\prime}, m^{\prime}}(a, s, u+1) T_{k, m}^{k^{\prime}, m^{\prime}}(a, s+1, u)}{T_{k, m+1}^{k^{\prime}, m^{\prime}}(a, s+1, u) T_{k, m}^{k^{\prime}, m^{\prime}}(a, s, u+1)}-e^{\partial_{u}-\partial_{s}} .
\end{aligned}
$$

The second rows of (18) can be similarly rewritten:

$$
\begin{aligned}
\Phi_{k+1, m}^{k^{\prime}, m^{\prime}}(a, s, u) & =\hat{\mathcal{C}}_{k, m}^{k^{\prime}, m^{\prime}}(a, s, u) \Phi_{k, m}^{k^{\prime}, m^{\prime}}(a, s, u), \\
\Phi_{k, m-1}^{k^{\prime}, m^{\prime}}(a, s, u) & =\hat{\mathcal{D}}_{k, m}^{k^{\prime}, m^{\prime}}(a, s, u) \Phi_{k, m}^{k^{\prime}, m^{\prime}}(a, s, u),
\end{aligned}
$$

where

$$
\Phi_{k, m}^{k^{\prime}, m^{\prime}}(a, s, u)=\frac{T_{k, m}^{k^{\prime}, m^{\prime}}(a, s+1, u)}{T_{k, m}^{k^{\prime}, m^{\prime}}(a+1, s, u)}
$$

and $\hat{\mathcal{C}}_{k, m}^{k^{\prime}, m^{\prime}}(a, s, u)$ and $\hat{\mathcal{D}}_{k, m}^{k^{\prime}, m^{\prime}}(a, s, u)$ are operators acting on the functions $\Phi_{k, m}^{k^{\prime}, m^{\prime}}(a, s, u)$ :

$$
\begin{aligned}
& \hat{\mathcal{C}}_{k, m}^{k^{\prime}, m^{\prime}}(a, s, u)=\frac{T_{k+1, m}^{k^{\prime}, m^{\prime}}(a, s, u-1) T_{k, m}^{k^{\prime}, m^{\prime}}(a+1, s, u)}{T_{k+1, m}^{k^{\prime}, m^{\prime}}(a+1, s, u) T_{k, m}^{k^{\prime}, m^{\prime}}(a, s, u-1)}+e^{-\partial_{u}-\partial_{a}}, \\
& \hat{\mathcal{D}}_{k, m}^{k^{\prime}, m^{\prime}}(a, s, u)=\frac{T_{k, m-1}^{k^{\prime}, m^{\prime}}(a, s, u-1) T_{k, m}^{k^{\prime}, m^{\prime}}(a+1, s, u)}{T_{k, m-1}^{k^{\prime}, m^{\prime}}(a+1, s, u) T_{k, m}^{k^{\prime}, m^{\prime}}(a, s, u-1)}+e^{-\partial_{u}-\partial_{a}} .
\end{aligned}
$$

In this form the (BT1) and (BT2) Bäcklund transformations appear as linear problems for the difference operators (30), (31), (35) and (36) with particular solutions (29) and (34). 
Equations (27) and (28) hold for any $k, m, k^{\prime}, m^{\prime}$, so the function $\Psi_{k-1, m+1}^{k^{\prime}, m^{\prime}}(a, s, u)$ can be represented in two different ways by subsequent action of operators (301), (31):

$$
\begin{array}{r}
\Psi_{k-1, m+1}^{k^{\prime}, m^{\prime}}(a, s, u) \equiv \hat{\mathcal{B}}_{k-1, m}^{k^{\prime}, m^{\prime}}(a, s, u) \hat{\mathcal{A}}_{k, m}^{k^{\prime}, m^{\prime}}(a, s, u) \Psi_{k, m}^{k^{\prime}, m^{\prime}}(a, s, u)= \\
\hat{\mathcal{A}}_{k, m+1}^{k^{\prime}, m^{\prime}}(a, s, u) \hat{\mathcal{B}}_{k, m}^{k^{\prime}, m^{\prime}}(a, s, u) \Psi_{k, m}^{k^{\prime}, m^{\prime}}(a, s, u) .
\end{array}
$$

Expanding both sides of (37) and canceling the identical terms, one ends up with a non-trivial relation connecting the T-functions with different $k$ and $m$. It takes the form

$$
\frac{T_{k, m}^{k^{\prime}, m^{\prime}}(a, s+1, u) T_{k+1, m+1}^{k^{\prime}, m^{\prime}}(a, s, u+1)-T_{k, m}^{k^{\prime}, m^{\prime}}(a, s, u+1) T_{k+1, m+1}^{k^{\prime}, m^{\prime}}(a, s+1, u)}{T_{k+1, m}^{k^{\prime}, m^{\prime}}(a, s, u+1) T_{k, m+1}^{k^{\prime}, m^{\prime}}(a, s+1, u)}=
$$

where $f_{k, m}^{k^{\prime}, m^{\prime}}(a, u+s)$ is an arbitrary function of its variables. Comparing (묘) with a similar equation obtained in the same way from the other pair of linear problems (32) and (33), one can see that $f_{k, m}^{k^{\prime}, m^{\prime}}$ depends only on the combination $u+s-a$ as well as on its indices. This function can be fixed by taking $s=m$ in (38) and exploiting the boundary conditions (24). This fixes the function $f_{k, m}^{k^{\prime}, m^{\prime}}(a, u+s)$ to be 1 .

Following a similar train of thoughts such TT-relations can be derived from the pair of linear problems (32) and (33). Thus the two main TT-relations coming from the right Bäcklund transformations (18) can be summarized as follows:

$$
\begin{aligned}
& \frac{T_{k, m}^{k^{\prime}, m^{\prime}}(a, s+1, u) T_{k+1, m+1}^{k^{\prime}, m^{\prime}}(a, s, u+1)-T_{k, m}^{k^{\prime}, m^{\prime}}(a, s, u+1) T_{k+1, m+1}^{k^{\prime}, m^{\prime}}(a, s+1, u)}{T_{k+1, m}^{k^{\prime}, m^{\prime}}(a, s, u+1) T_{k, m+1}^{k^{\prime}, m^{\prime}}(a, s+1, u)}=1, \\
& \frac{T_{k, m}^{k^{\prime}, m^{\prime}}(a-1, s, u) T_{k+1, m+1}^{k^{\prime}, m^{\prime}}(a, s, u+1)-T_{k, m}^{k^{\prime}, m^{\prime}}(a, s, u+1) T_{k+1, m+1}^{k^{\prime}, m^{\prime}}(a-1, s, u)}{T_{k+1, m}^{k^{\prime}, m^{\prime}}(a, s, u+1) T_{k, m+1}^{k^{\prime}, m^{\prime}}(a-1, s, u)}=1 .
\end{aligned}
$$

We note that as it was done in [39] further bilinear TT-relations can be derived from (18), which will not be presented here.

With the help of the method described above very similar TT-relations can be obtained from the left Bäcklund transformations (21). The two most important of 
them read as:

$$
\begin{aligned}
& \frac{T_{k, m}^{k^{\prime}, m^{\prime}}(a, s-1, u) T_{k, m}^{k^{\prime}+1, m^{\prime}+1}(a, s, u+1)-T_{k, m}^{k^{\prime}, m^{\prime}}(a, s, u+1) T_{k, m}^{k^{\prime}+1, m^{\prime}+1}(a, s-1, u)}{T_{k, m}^{k^{\prime}+1, m^{\prime}}(a, s, u+1) T_{k, m}^{k^{\prime}, m^{\prime}+1}(a, s-1, u)}=1, \\
& \frac{T_{k, m}^{k^{\prime}, m^{\prime}}(a-1, s, u) T_{k, m}^{k^{\prime}+1, m^{\prime}+1}(a, s, u+1)-T_{k, m}^{k^{\prime}, m^{\prime}}(a, s, u+1) T_{k, m}^{k^{\prime}+1, m^{\prime}+1}(a-1, s, u)}{T_{k, m}^{k^{\prime}+1, m^{\prime}}(a, s, u+1) T_{k, m}^{k^{\prime}, m^{\prime}+1}(a-1, s, u)}=1 .
\end{aligned}
$$

The so-called QQ-relations are obtained by restricting TT-relations (39,42) to the boundary of the T-shaped fat hook. The most important ones can be derived by taking (39) and (41) along the line $(0, s)$. The QQ-relations for the left and right moving functions $\left(Q^{k^{\prime}, m^{\prime}}\right.$ and $\left.\tilde{Q}_{k, m}\right)$ of the lower boundary line of the T-shaped fathook respectively admit formally the same QQ-relations as the those of the $g l(K \mid M)$ super spin chains [31, 39]:

$$
\begin{gathered}
\tilde{Q}_{k, m}(u) \tilde{Q}_{k+1, m+1}(u+2)-\tilde{Q}_{k, m}(u+2) \tilde{Q}_{k+1, m+1}(u)=\tilde{Q}_{k, m+1}(u) \tilde{Q}_{k+1, m}(u+2),(43) \\
Q^{k^{\prime}, m^{\prime}}(u) Q^{k^{\prime}+1, m^{\prime}+1}(u+2)-Q^{k^{\prime}, m^{\prime}}(u+2) Q^{k^{\prime}+1, m^{\prime}+1}(u)=Q^{k^{\prime}, m^{\prime}+1}(u) Q^{k^{\prime}+1, m^{\prime}}(u+2) .
\end{gathered}
$$

Another QQ-relation can be obtained by taking (41) along the line $(k, s)$ for $s \geq m$ :

$$
\tilde{\mathcal{Q}}_{k}^{k^{\prime}, m^{\prime}}(u) \tilde{\mathcal{Q}}_{k}^{k^{\prime}+1, m^{\prime}+1}(u+2)-\tilde{\mathcal{Q}}_{k}^{k^{\prime}, m^{\prime}}(u+2) \tilde{\mathcal{Q}}_{k}^{k^{\prime}+1, m^{\prime}+1}(u)=\tilde{\mathcal{Q}}_{k}^{k^{\prime}, m^{\prime}+1}(u) \tilde{\mathcal{Q}}_{k}^{k^{\prime}+1, m^{\prime}}(u+2) .
$$

Finally restricting equations (39) to the line $\left(k^{\prime}, s\right)$ for $s \leq-m^{\prime}$ one ends up with an additional type of QQ-relation:

$$
\mathcal{Q}_{k, m}^{k^{\prime}}(u) \mathcal{Q}_{k+1, m+1}^{k^{\prime}}(u+2)-\mathcal{Q}_{k, m}^{k^{\prime}}(u+2) \mathcal{Q}_{k+1, m+1}^{k^{\prime}}(u)=\mathcal{Q}_{k, m+1}^{k^{\prime}}(u) \mathcal{Q}_{k+1, m}^{k^{\prime}}(u+2)
$$

In case of $g l(K \mid M)$ super spin chains [39] QQ-relations such as (43,46) were used to derive Bethe equations for the spin chain. This was possible because in case of spin chains, based on earlier experience with algebraic Bethe ansatz, it is assumed that the Q-functions are polynomial functions of $u$, and then QQ-relations allow one to derive a closed set of equations for their zeroes. The zeroes of Q-functions are the Bethe roots which determine uniquely the Q-functions. Then the T-functions can be expressed in terms of the Q-functions, which provide the solution of the Hirota equations. 
In our case, for the T-shaped fat-hook, we do not use QQ-relations to get Bethe equations, because we do not assume anything about the functional form of the Q-functions. We consider them as general functions as possible, which means that they can be arbitrary functions modulo equations which connect them (for example QQ-relations). Our purpose is only to express T-functions in terms of Q-functions, which is presented in the next section. In this way we can parameterize the infinitely many T-functions in terms of a few arbitrarily chosen functions.

In view of future applications these functions have to be dressed by some analytic properties, so that one can derive a set of nonlinear integral equations (NLIE 1 ) governing the exact planar spectrum of AdS/CFT.

\section{Integration of the Hirota equations}

In this section following the lines of [39] we develop a general algorithm to solve the Hirota equations expressing the functions $T_{k, m}^{k^{\prime}, m^{\prime}}(a, s, u)$ in terms of boundary functions $Q^{k^{\prime}, m^{\prime}}(u)$ and $\tilde{Q}_{k, m}(u)$.

The starting point is an alternative representation of the first and second rows of equations (18) and (21). The equations coming from (18) are formulated by shift operators, which change only the lower indices of $T_{k, m}^{k^{\prime}, m^{\prime}}(a, s, u)$ :

$$
\begin{aligned}
& T_{k-1, m}^{k^{\prime}, m^{\prime}}(a, s, u)=\hat{H}_{k-, m}^{k^{\prime}, m^{\prime}}(a-1, s, u) T_{k, m}^{k^{\prime}, m^{\prime}}(a, s, u), \\
& T_{k, m+1}^{k^{\prime}, m^{\prime}}(a, s, u)=\hat{H}_{k, m+}^{k^{\prime}, m^{\prime}}(a-1, s, u) T_{k, m}^{k^{\prime}, m^{\prime}}(a, s, u), \\
& T_{k+1, m}^{k^{\prime}, m^{\prime}}(a, s, u)=\hat{H}_{k+, m}^{k^{\prime}, m^{\prime}}(a, s-1, u) T_{k, m}^{k^{\prime}, m^{\prime}}(a, s, u), \\
& T_{k, m-1}^{k^{\prime}, m^{\prime}}(a, s, u)=\hat{H}_{k, m-}^{k^{\prime}, m^{\prime}}(a, s-1, u) T_{k, m}^{k^{\prime}, m^{\prime}}(a, s, u),
\end{aligned}
$$

where the "right shift operators" 2 take the form:

$$
\hat{H}_{k-, m}^{k^{\prime}, m^{\prime}}(a, s, u)=\frac{T_{k-1, m}^{k^{\prime}, m^{\prime}}(a, s, u+1)}{T_{k, m}^{k^{\prime}, m^{\prime}}(a, s, u+1)}-\frac{T_{k-1, m}^{k^{\prime}, m^{\prime}}(a, s+1, u)}{T_{k, m}^{k^{\prime}, m^{\prime}}(a, s, u+1)} e^{\partial_{u}-\partial_{s}},
$$

\footnotetext{
${ }^{1}$ The nonlinear integral equation technique (NLIE) proved to be the most efficient tool for describing the finite size effects in spin chains and relativistic quantum field theories. So, this is the expectation for the AdS/CFT as well.

${ }^{2}$ We call these shift operators "right shift operators", because they change the indices $k$ and $m$, which are the coordinates of the corner point on the right hand side of the T-shaped fat-hook. The origin of the term "left shift operator" is analogous.
} 


$$
\begin{gathered}
\hat{H}_{k, m+}^{k^{\prime}, m^{\prime}}(a, s, u)=\frac{T_{k, m+1}^{k^{\prime}, m^{\prime}}(a, s, u+1)}{T_{k, m}^{k^{\prime}, m^{\prime}}(a, s, u+1)}-\frac{T_{k, m+1}^{k^{\prime}, m^{\prime}}(a, s+1, u)}{T_{k, m}^{k^{\prime}, m^{\prime}}(a, s, u+1)} e^{\partial_{u}-\partial_{s}}, \\
\hat{H}_{k+, m}^{k^{\prime}, m^{\prime}}(a, s, u)=\frac{T_{k+1, m}^{k^{\prime}, m^{\prime}}(a, s, u-1)}{T_{k, m}^{k^{\prime}, m^{\prime}}(a, s, u-1)}+\frac{T_{k+1, m}^{k^{\prime}, m^{\prime}}(a+1, s, u)}{T_{k, m}^{k^{\prime}, m^{\prime}}(a, s, u-1)} e^{-\partial_{u}-\partial_{a}}, \\
\hat{H}_{k, m-}^{k^{\prime}, m^{\prime}}(a, s, u)=\frac{T_{k, m-1}^{k^{\prime}, m^{\prime}}(a, s, u-1)}{T_{k, m}^{k^{\prime}, m^{\prime}}(a, s, u-1)}+\frac{T_{k, m-1}^{k^{\prime}, m^{\prime}}(a+1, s, u)}{T_{k, m}^{k^{\prime}, m^{\prime}}(a, s, u-1)} e^{-\partial_{u}-\partial_{a}} .
\end{gathered}
$$

The equations coming from (21) are formulated by shift operators shifting only the upper indices of $T_{k, m}^{k^{\prime}, m^{\prime}}(a, s, u)$ :

$$
\begin{aligned}
& T_{k, m}^{k^{\prime}-1, m^{\prime}}(a, s, u)=\hat{G}_{k, m}^{k^{\prime}-, m^{\prime}}(a-1, s, u) T_{k, m}^{k^{\prime}, m^{\prime}}(a, s, u), \\
& T_{k, m}^{k^{\prime}, m^{\prime}+1}(a, s, u)=\hat{G}_{k, m}^{k^{\prime}, m^{\prime}+}(a-1, s, u) T_{k, m}^{k^{\prime}, m^{\prime}}(a, s, u), \\
& T_{k, m}^{k^{\prime}+1, m^{\prime}}(a, s, u)=\hat{G}_{k, m}^{k^{\prime}+m^{\prime}}(a, s+1, u) T_{k, m}^{k^{\prime}, m^{\prime}}(a, s, u), \\
& T_{k, m}^{k^{\prime}, m^{\prime}-1}(a, s, u)=\hat{G}_{k, m}^{k^{\prime}, m^{\prime}-}(a, s+1, u) T_{k, m}^{k^{\prime}, m^{\prime}}(a, s, u),
\end{aligned}
$$

where the "left shift operators" take the form:

$$
\begin{gathered}
\hat{G}_{k, m}^{k^{\prime}-, m^{\prime}}(a, s, u)=\frac{T_{k, m}^{k^{\prime}-1, m^{\prime}}(a, s, u+1)}{T_{k, m}^{k^{\prime}, m^{\prime}}(a, s, u+1)}-\frac{T_{k, m}^{k^{\prime}-1, m^{\prime}}(a, s-1, u)}{T_{k, m}^{k^{\prime}, m^{\prime}}(a, s, u+1)} e^{\partial_{u}+\partial_{s},} \\
\hat{G}_{k, m}^{k^{\prime}, m^{\prime}+}(a, s, u)=\frac{T_{k, m}^{k^{\prime}, m^{\prime}+1}(a, s, u+1)}{T_{k, m}^{k^{\prime}, m^{\prime}}(a, s, u+1)}-\frac{T_{k, m}^{k^{\prime}, m^{\prime}+1}(a, s-1, u)}{T_{k, m}^{k^{\prime}, m^{\prime}}(a, s, u+1)} e^{\partial_{u}+\partial_{s}}, \\
\hat{G}_{k, m}^{k^{\prime}+, m^{\prime}}(a, s, u)=\frac{T_{k, m}^{k^{\prime}+1, m^{\prime}}(a, s, u-1)}{T_{k, m}^{k^{\prime}, m^{\prime}}(a, s, u-1)}+\frac{T_{k, m}^{k^{\prime}+1, m^{\prime}}(a+1, s, u)}{T_{k, m}^{k^{\prime}, m^{\prime}}(a, s, u-1)} e^{-\partial_{u}-\partial_{a}}, \\
\hat{G}_{k, m}^{k^{\prime}, m^{\prime}-}(a, s, u)=\frac{T_{k, m}^{k^{\prime}, m^{\prime}-1}(a, s, u-1)}{T_{k, m}^{k^{\prime}, m^{\prime}}(a, s, u-1)}+\frac{T_{k, m}^{k^{\prime}, m^{\prime}-1}(a+1, s, u)}{T_{k, m}^{k^{\prime}, m^{\prime}}(a, s, u-1)} e^{-\partial_{u}-\partial_{a}} .
\end{gathered}
$$

Starting from $T_{k, m}^{k^{\prime}, m^{\prime}}$, with the help of shift operators, the $T_{k \mp 1, m \pm 1}^{k^{\prime}, m^{\prime}}$ and $T_{k, m}^{k^{\prime} \mp 1, m^{\prime} \pm 1}$ functions can be obtained in two different ways, implying "weak" zero curvature conditions for the shift operators:

$$
\begin{gathered}
T_{k \mp 1, m \pm 1}^{k^{\prime}, m^{\prime}}=\hat{H}_{k \mp 1, m \pm}^{k^{\prime}, m^{\prime}} \hat{H}_{k \mp, m}^{k^{\prime}, m^{\prime}} T_{k, m}^{k^{\prime}, m^{\prime}}=\hat{H}_{k \mp, m \pm 1}^{k^{\prime}, m^{\prime}} \hat{H}_{k, m \pm}^{k^{\prime}, m^{\prime}} T_{k, m}^{k^{\prime}, m^{\prime}}, \\
T_{k, m}^{k^{\prime} \mp 1, m^{\prime} \pm 1}=\hat{G}_{k, m}^{k^{\prime} \mp 1, m^{\prime} \pm} \hat{G}_{k, m}^{k^{\prime} \mp, m^{\prime}} T_{k, m}^{k^{\prime}, m^{\prime}}=\hat{G}_{k, m}^{k^{\prime} \mp, m^{\prime} \pm 1} \hat{G}_{k, m}^{k^{\prime}, m^{\prime} \pm} T_{k, m}^{k^{\prime}, m^{\prime}}
\end{gathered}
$$


where for short we skipped the arguments $(a-1, s, u)$ for the shift operators and $(a, s, u)$ for the T-functions. Equations (63) and (64) are termed "weak" zero curvature conditions, because they are satisfied only when the shift operators act on the functions $T_{k, m}^{k^{\prime}, m^{\prime}}$. Nevertheless, exploiting the TT-relations (39-42), it can be shown that the "weak" zero curvature conditions imply the strong operator form as well:

$$
\begin{aligned}
\hat{H}_{k \mp 1, m \pm}^{k^{\prime}, m^{\prime}}(a, s, u) \hat{H}_{k \mp, m}^{k^{\prime}, m^{\prime}}(a, s, u) & =\hat{H}_{k \mp, m \pm 1}^{k^{\prime}, m^{\prime}}(a, s, u) \hat{H}_{k, m \pm}^{k^{\prime}, m^{\prime}}(a, s, u), \\
\hat{G}_{k, m}^{k^{\prime} \mp 1, m^{\prime} \pm}(a, s, u) \hat{G}_{k, m}^{k^{\prime} \mp, m^{\prime}}(a, s, u) & =\hat{G}_{k, m}^{k^{\prime} \mp, m^{\prime} \pm 1}(a, s, u) \hat{G}_{k, m}^{k^{\prime}, m^{\prime} \pm}(a, s, u) .
\end{aligned}
$$

Having the above relations at hand we express the T-functions in terms of the $Q^{k^{\prime}, m^{\prime}}$ and $\tilde{Q}_{k, m}$ boundary functions. To avoid problems coming from division by zero in the shift operators, we determine the T-functions along the infinite line $(1, s)$ and then T-functions for other values of $a$ and $s$ can be determined by the application of the Bazhanov-Reshetikhin formula [43, 30]:

$$
\tilde{T}_{k, m}^{k^{\prime}, m^{\prime}}(a, s, u)=\operatorname{det}_{1 \leq i, j \leq a} \tilde{T}_{k, m}^{k^{\prime}, m^{\prime}}(1, s+i-j, u+a+1-i-j)
$$

where

$$
\tilde{T}_{k, m}^{k^{\prime}, m^{\prime}}(a, s, u)=\frac{T_{k, m}^{k^{\prime}, m^{\prime}}(a, s, u)}{Q^{k^{\prime}, m^{\prime}}(u+a+s) \tilde{Q}_{k, m}(u-a-s)} .
$$

To express $T_{k, m}^{k^{\prime}, m^{\prime}}(1, s, u)$ in terms of $Q^{k^{\prime}, m^{\prime}}$ and $\tilde{Q}_{k, m}$, the equations (151), (152) and (59), (60) have to be taken at $a=1$. In this case the corresponding shift operators depend only on the boundary functions $Q^{k^{\prime}, m^{\prime}}$ and $\tilde{Q}_{k, m}$. Moreover it turns out that for $a=0$ the right shift operators depend only on the lower indices $k$ and $m$, while the left shift operators depend only on the upper indices $k^{\prime}$ and $m^{\prime}$. This is why we delete the irrelevant pair of indices from them. The relevant shift operators are of the form:

$$
\begin{gathered}
\hat{H}_{k-, m}(0, s, u)=\frac{\tilde{Q}_{k-1, m}(u-s+1)}{\tilde{Q}_{k, m}(u-s+1)}-\frac{\tilde{Q}_{k-1, m}(u-s-1)}{\tilde{Q}_{k, m}(u-s+1)} e^{\partial_{u}-\partial_{s}}, \\
\hat{H}_{k, m+}(0, s, u)=\frac{\tilde{Q}_{k, m+1}(u-s+1)}{\tilde{Q}_{k, m}(u-s+1)}-\frac{\tilde{Q}_{k, m+1}(u-s-1)}{\tilde{Q}_{k, m}(u-s+1)} e^{\partial_{u}-\partial_{s}}, \\
\hat{G}^{k^{\prime}-, m^{\prime}}(0, s, u)=\frac{Q^{k^{\prime}-1, m^{\prime}}(u+s+1)}{Q^{k^{\prime}, m^{\prime}}(u+s+1)}-\frac{Q^{k^{\prime}-1, m^{\prime}}(u+s-1)}{Q^{k^{\prime}, m^{\prime}}(u+s+1)} e^{\partial_{u}+\partial_{s}},
\end{gathered}
$$




$$
\hat{G}^{k^{\prime}, m^{\prime}+}(0, s, u)=\frac{Q^{k^{\prime}, m^{\prime}+1}(u+s+1)}{Q^{k^{\prime}, m^{\prime}}(u+s+1)}-\frac{Q^{k^{\prime}, m^{\prime}+1}(u+s-1)}{Q^{k^{\prime}, m^{\prime}}(u+s+1)} e^{\partial_{u}+\partial_{s}} .
$$

It is important to recognize that the left shift operators depend only on $u+s$ and the right ones on $u-s$, which implies that along this special line the left and right shift operators commute for any indices:

$$
\hat{G}^{\alpha}(0, s, u) \hat{H}_{\beta}(0, s, u)=\hat{H}_{\beta}(0, s, u) \hat{G}^{\alpha}(0, s, u),
$$

where $\alpha$ denotes either the pair of indices " $k^{\prime}, m^{\prime}+"$ or " $k^{\prime}-, m^{\prime \prime}$, while similarly $\beta$ is either for the pair of indices " $k, m+$ " or " $k-, m$ ".

Relations (73) ensure that during the undressing procedure the size of the system on the left and right hand sides can be decreased independently. Moreover equations (65) and (66) ensure that the different orders of decreasing the values of $k, m$ and $k^{\prime}, m^{\prime}$ respectively lead to the same final result.

Thus to determine an undressing path, one can define two arbitrary oriented zigzag paths; one going from $(0,0)$ to $\left(K^{\prime},-M^{\prime}\right)$ denoted by $\gamma^{K^{\prime}, M^{\prime}}$, and the other $\gamma_{K, M}$ goes from $(0,0)$ to $(K, M)$. These two paths are characterized by their oriented edges, in such a way that to each edge a pair of vectors $(\mathbf{x}, \mathbf{n})$ is assigned, where $\mathbf{x}$ denotes the starting point of the oriented edge on the $(a, s)$ lattice, while $\mathbf{n}$ is a unit vector pointing from $\mathbf{x}$ to the endpoint of the edge. For the path $\gamma^{K^{\prime}, M^{\prime}}$ the edge characterizing pairs of vectors are denoted by $\left(\mathbf{x}_{\mathbf{L}}, \mathbf{n}_{\mathbf{L}}\right)$, while for $\gamma_{K, M}$ they are denoted by $\left(\mathbf{x}_{\mathbf{R}}, \mathbf{n}_{\mathbf{R}}\right)$. The unit vectors $\mathbf{n}_{\mathbf{L}}$ can take values $(1,0)$ or $(0,-1)$, while the possible choices for $\mathbf{n}_{\mathbf{R}}$ are $(1,0)$ or $(0,1)$.

Using the previous characterization, inverse undressing operators 3 can be assigned to the edges of the paths. They are as follows:

$$
\begin{aligned}
& \hat{V}_{\left(\mathbf{x}_{\mathbf{R}}, \mathbf{n}_{\mathbf{R}}\right)}^{R}(s, u)=\left\{\begin{array}{cc}
\hat{H}_{k, m+}(0, s, u), & \mathbf{x}_{\mathbf{R}}=(k, m), \quad \mathbf{n}_{\mathbf{R}}=(0,1) \\
\hat{H}_{(k+1)-, m}^{-1}(0, s, u), & \mathbf{x}_{\mathbf{R}}=(k, m), \quad \mathbf{n}_{\mathbf{R}}=(1,0),
\end{array}\right. \\
& \hat{V}_{\left(\mathbf{x}_{\mathbf{L}}, \mathbf{n}_{\mathbf{L}}\right)}^{L}(s, u)=\left\{\begin{array}{c}
\hat{G}^{k^{\prime}, m^{\prime}+}(0, s, u), \quad \mathbf{x}_{\mathbf{L}}=\left(k^{\prime},-m^{\prime}\right), \quad \mathbf{n}_{\mathbf{L}}=(0,-1) \\
\left(\hat{G}^{\left(k^{\prime}+1\right)-, m^{\prime}}(0, s, u)\right)^{-1}, \quad \mathbf{x}_{\mathbf{L}}=\left(k^{\prime},-m^{\prime}\right), \quad \mathbf{n}_{\mathbf{L}}=(1,0),
\end{array}\right.
\end{aligned}
$$

\footnotetext{
${ }^{3}$ These operators are called "inverse undressing operators", because they realize the inverse of the undressing procedure, which means that their successive application to the trivial element $T_{0,0}^{0,0}(a, s, u)$ of the hierarchy of T-functions provides the solution to the original problem.
} 
where $\hat{V}_{\left(\mathbf{x}_{\mathbf{L}}, \mathbf{n}_{\mathbf{L}}\right)}^{L}(s, u)$ is assigned to the edge $\left(\mathbf{x}_{\mathbf{L}}, \mathbf{n}_{\mathbf{L}}\right)$ of $\gamma^{K^{\prime}, M^{\prime}}$, while $\hat{V}_{\left(\mathbf{x}_{\mathbf{R}}, \mathbf{n}_{\mathbf{R}}\right)}^{R}(s, u)$ is assigned to the edge $\left(\mathbf{x}_{\mathbf{R}}, \mathbf{n}_{\mathbf{R}}\right)$ of $\gamma_{K, M}$. In equations (174) and (75) the inverse operators of $\hat{H}_{(k+1)-, m}(0, s, u)$ and $\hat{G}^{\left(k^{\prime}+1\right)-, m^{\prime}}(0, s, u)$ appear. It can be shown [39] that these operators have nontrivial kernels. Namely, the kernel of $\hat{H}_{k-, m}(0, s, u)$ consists of functions of the form $\tilde{Q}_{k-1, m}(u-s-1) \tilde{f}(u+s)$ and the kernel of $\hat{G}^{k^{\prime}-, m^{\prime}}(0, s, u)$ consists of functions of the form $Q^{k^{\prime}-1, m}(u+s-1) f(u-s)$, with $f(u)$ and $\tilde{f}(u)$ being arbitrary functions. In formulae (74) and (75) the inverses of $\hat{H}_{k-, m}(0, s, u)$ and $\hat{G}^{k^{\prime}-m^{\prime}}(0, s, u)$ are meant modulo these kernels.

Following from equations (47), (48) (마), (156) taken at $a=1$, the T-functions $T_{K, M}^{K^{\prime}, M^{\prime}}(1, s, u)$ can be obtained as ordered products of operators (74) and (75) acting on $T_{0,0}^{0,0}(1, s, u)$ :

$$
T_{K, M}^{K^{\prime}, M^{\prime}}(1, s, u)=\hat{\mathcal{V}}_{L}(s, u) \hat{\mathcal{V}}_{R}(s, u) T_{0,0}^{0,0}(1, s, u),
$$

where the operators $\hat{\mathcal{V}}_{L}(s, u)$ and $\hat{\mathcal{V}}_{R}(s, u)$ commute due to (73), and they correspond to paths $\gamma^{K^{\prime}, M^{\prime}}$ and $\gamma_{K, M}$ respectively. They read as

$$
\begin{aligned}
& \hat{\mathcal{V}}_{L}(s, u)=\prod_{\left(\mathbf{x}_{\mathbf{L}}, \mathbf{n}_{\mathbf{L}}\right) \in \gamma^{K^{\prime}, M^{\prime}}} \hat{V}_{\left(\mathbf{x}_{\mathbf{L}}, \mathbf{n}_{\mathbf{L}}\right)}^{L}(s, u), \\
& \hat{\mathcal{V}}_{R}(s, u)=\prod_{\left(\mathbf{x}_{\mathbf{R}}, \mathbf{n}_{\mathbf{R}}\right) \in \gamma_{K, M}}^{\rightarrow} \hat{V}_{\left(\mathbf{x}_{\mathbf{R}}, \mathbf{n}_{\mathbf{R}}\right)}^{R}(s, u),
\end{aligned}
$$

where the arrow means that ordered product of the operators has to be taken, in such a way that operators on the right hand side of (77) and (78) have to be ordered from the last edge of the corresponding path (ending at the origin). I.e. reading the product of operators on the right hand side of (77) and (78) from the left to the right, the first operator corresponds to the last edge of the oriented paths $\gamma^{K^{\prime}, M^{\prime}}$ and $\gamma_{K, M}$ respectively. The T-function $T_{0,0}^{0,0}(1, s, u)$ corresponds to a trivial domain, thus it can be trivially expressed by the boundary functions (24):

$$
T_{0,0}^{0,0}(1, s, u)=Q^{0,0}(u+1) \tilde{Q}_{0,0}(u-1) \delta_{s, 0}=Q^{0,0}(u-1) \tilde{Q}_{0,0}(u+1) \delta_{s, 0} .
$$

The formulae (76)-(179) provide the solution of the Hirota equations defined on a T-shaped fat-hook of type $\left(K^{\prime}, M^{\prime}\right) \odot(K, M)$, in such a way that the T-functions are expressed in terms of the boundary functions (Q-functions) of the hierarchy of Hirota equations defined by our chain of Bäcklund transformations. 


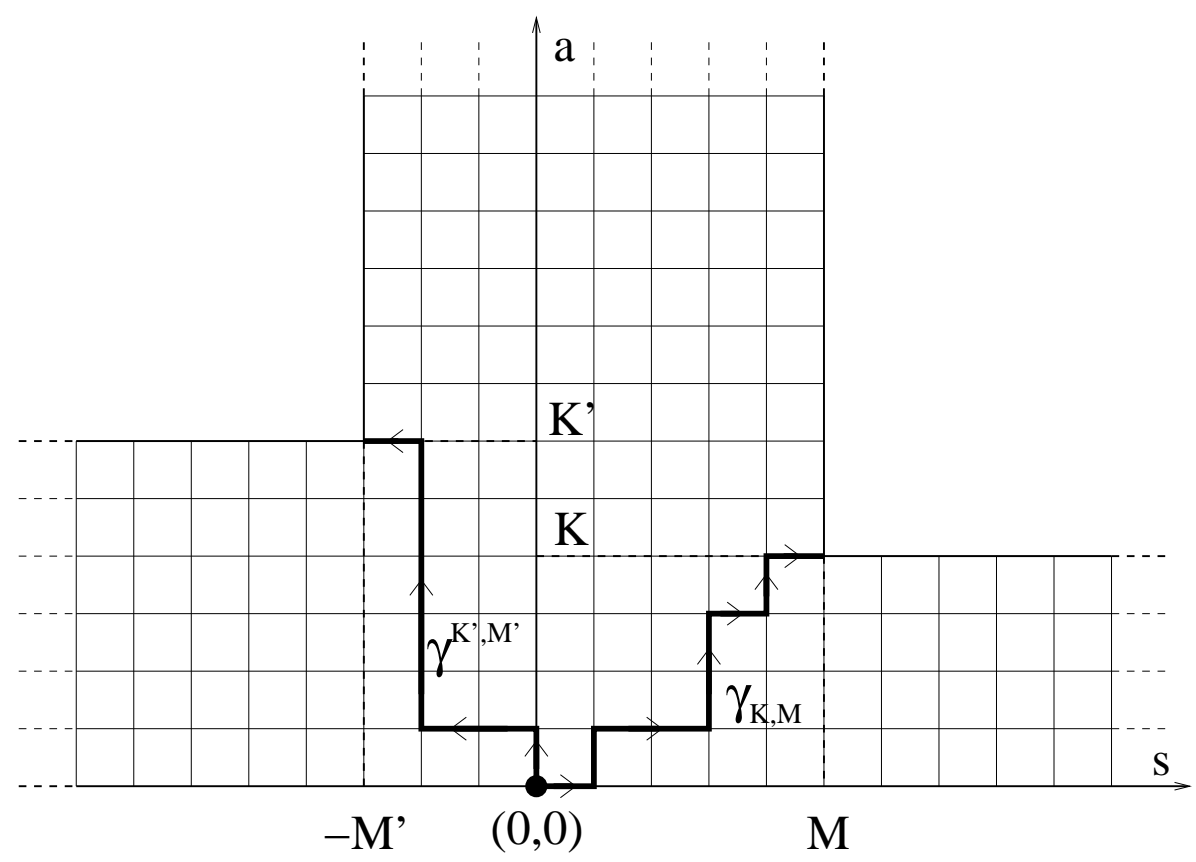

Figure 6: Undressing paths for a T-shaped fat-hook of type $\left(K^{\prime}, M^{\prime}\right) \odot(K, M)$.

The application of formula (76) to the case of AdS/CFT (i.e. $K=M=K^{\prime}=$ $M^{\prime}=2$ ) will be presented in the next section. One remark concerning equation (76) is in order. It is not true that the functions $T_{K, M}^{K^{\prime}, M^{\prime}}(1, s, u)$ are products of eigenvalues of a $g l(K \mid M)$ and a $g l\left(K^{\prime} \mid M^{\prime}\right)$ transfer matrices, but rather they can be obtained by acting a $g l(K \mid M)$ type $\left(\hat{\mathcal{V}}_{R}\right)$ and a $g l\left(K^{\prime} \mid M^{\prime}\right)$ type $\left(\hat{\mathcal{V}}_{L}\right)$ generating series on $T_{0,0}^{0,0}(1, s, u)$. This fact accounts for a possible $G L(K \mid M) \otimes G L\left(K^{\prime} \mid M^{\prime}\right)$ symmetry 4 behind the T-system defined on a T-shaped fat-hook of type $\left(K^{\prime}, M^{\prime}\right) \odot(K, M)$.

The section is closed with a remark concerning the arbitraryness of the undressing procedure. In the undressing procedure in addition to the arbitrary choice of the paths $\gamma^{K^{\prime}, M^{\prime}}$ and $\gamma_{K, M}$ there is an additional arbitraryness. Namely the origin of our state of reference on the $(a, s)$ lattice can be shifted freely within the interval $\left[-M^{\prime}, M\right]$. This means, that for example the T-shaped fat-hook with corner point coordinates $\left(K^{\prime},-M^{\prime}\right)$ and $(K, M)$ is equivalent to T-shaped fat-hooks with corner point coordinates $\left(K^{\prime},-M^{\prime}+m\right)$ and $(K, M+m)$, where $-M \leq m \leq M^{\prime}$. (We

\footnotetext{
${ }^{4}$ In this remark the symmetry means that this T-system possibly correspond to the TBA equations of an appropriate $G L(K \mid M) \otimes G L\left(K^{\prime} \mid M^{\prime}\right)$ invariant scattering theory.
} 
restricted the possible values for $m$, which is the origin shifting parameter, so that the origin will remain between the "s-coordinates" of the corner points.) This means that the number of zigzag paths defining the undressing procedure is:

$$
N_{\text {zigzag }}=\sum_{j=0}^{M+M^{\prime}}\left(\begin{array}{c}
K^{\prime}+M+M^{\prime}-j \\
K^{\prime}
\end{array}\right)\left(\begin{array}{c}
K+j \\
K
\end{array}\right)
$$

where $\left(\begin{array}{c}n \\ m\end{array}\right)$ stands for the binomial coefficient. In case of $\operatorname{AdS} / \mathrm{CFT}\left(K=K^{\prime}=\right.$ $\left.M=M^{\prime}=2\right)$ this counting gives $N_{z i g z a g}^{A d S}=\left(\begin{array}{l}9 \\ 5\end{array}\right)$ for the total number of possible undressing paths.

However it has to be noted that there may be such distinguished axes on the $(a, s)$ lattice, which distinguish certain choices for the origin. In case of AdS/CFT the analytic properties of Y-functions distinguish the vertical symmetry axis of the T-shaped fat-hook of type $(2,2) \odot(2,2)$. This is why we solve the AdS/CFT case from a state of reference, where the corner point coordinates of the T-shaped fathook are $(2,-2)$ and $(2,2)$ on the $(a, s)$ lattice. In this case the distinguished vertical symmetry axis of the T-shaped fat-hook is a vertical line starting from the origin. This choice for the origin is made, so that none of the zigzag paths will cross the distinguished axis.

\section{The AdS/CFT case}

In this section the method explained in the previous sections is applied to the case of AdS/CFT. Based on TBA calculations [24, 25] the T-system (2) of AdS/CFT is defined on a T-shaped fat-hook of type $(2,2) \odot(2,2)$ as a consequence of the $S U(2 \mid 2) \otimes S U(2 \mid 2)$ symmetry of the problem. Here we make special choices for the undressing paths $\gamma^{K^{\prime}, M^{\prime}}$ and $\gamma_{K, M}$ drawn in figure 7., and then we express the T-functions of the problem along the line $(1, s)$ in terms of the boundary functions $Q^{k^{\prime}, m^{\prime}}$ and $\tilde{Q}_{k, m}$. The generating series corresponding to the path $\gamma_{K, M}$ takes the form:

$$
\hat{\mathcal{V}}_{R}(s, u)=\hat{H}_{2,1+}(0, s, u) \hat{H}_{2-, 1}^{-1}(0, s, u) \hat{H}_{1-, 1}^{-1}(0, s, u) \hat{H}_{0,0+}(0, s, u)
$$




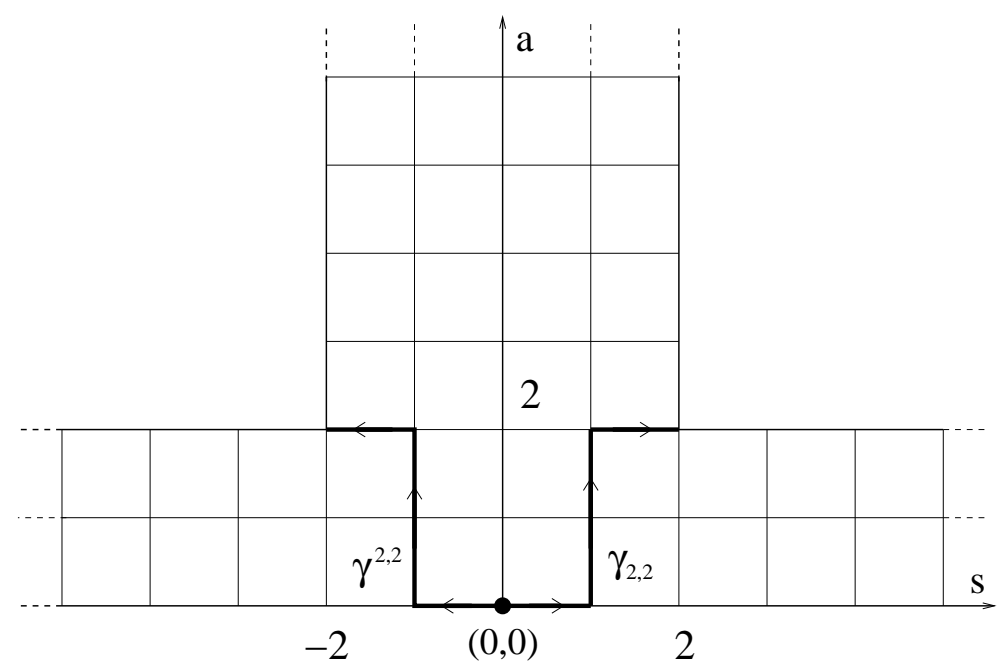

Figure 7: A possible undressing path for AdS/CFT.

while the generating series for $\gamma^{K^{\prime}, M^{\prime}}$ reads as:

$$
\hat{\mathcal{V}}_{L}(s, u)=\hat{G}^{2,1+}(0, s, u)\left(\hat{G}^{2-, 1}(0, s, u)\right)^{-1}\left(\hat{G}^{1-, 1}(0, s, u)\right)^{-1} \hat{G}^{0,0+}(0, s, u) .
$$

Expanding the inverse operators with respect to shift operators in $u$ and $s$ and collecting them to the right hand side of the expressions, one obtains the following result for the generating series $\hat{\mathcal{V}}_{L}(s, u)$ and $\hat{\mathcal{V}}_{R}(s, u)$ :

$$
\hat{\mathcal{V}}_{\Omega}(s, u)=\sum_{k=0}^{\infty} F_{\Omega}^{(k)}(u+\sigma s) e^{k\left(\partial_{u}+\sigma \partial_{s}\right)}, \quad \sigma=\left\{\begin{array}{cc}
+1 & \Omega=L \\
-1 & \Omega=R
\end{array}\right.
$$

where $\Omega$ stands for $L$ or $R$ and $\sigma$ can take values \pm 1 depending on the value of lower index $\left(L\right.$ or $R$ ) of the generating series $\hat{\mathcal{V}}_{\Omega}(s, u)$. The coefficients in (83) are of the 
form:

$$
\begin{array}{r}
F_{\Omega}^{(k)}(u)=E_{\Omega}(u) \sum_{j=0}^{k} A_{\Omega}(u+2(k-j)) B_{\Omega}(u+2 k)- \\
\Theta(k-1) E_{\Omega}(u) \sum_{j=0}^{k-1} A_{\Omega}(u+2(k-j-1)) C_{\Omega}(u+2(k-1))- \\
\Theta(k-1) F_{\Omega}(u) \sum_{j=0}^{k-1} A_{\Omega}(u+2(k-j)) B_{\Omega}(u+2 k)+ \\
\Theta(k-2) F_{\Omega}(u) \sum_{j=0}^{k-2} A_{\Omega}(u+2(k-j-1)) C_{\Omega}(u+2(k-1)),
\end{array}
$$

where the $A_{\Omega}, B_{\Omega}, C_{\Omega}, E_{\Omega}, F_{\Omega}$ coefficient functions can be directly expressed by the boundary functions of the problem:

$$
\begin{gathered}
A_{\Omega}(u)=\frac{\mathbf{Q}_{\Omega}^{2,1}(u+1) \mathbf{Q}_{\Omega}^{0,1}(u-1)}{\mathbf{Q}_{\Omega}^{1,1}(u+1) \mathbf{Q}_{\Omega}^{1,1}(u-1)}, \quad B_{\Omega}(u)=\frac{\mathbf{Q}_{\Omega}^{1,1}(u+1)}{\mathbf{Q}_{\Omega}^{0,1}(u-1) \mathbf{Q}_{\Omega}^{0,0}(u+1)}, \\
C_{\Omega}(u)=\frac{\mathbf{Q}_{\Omega}^{1,1}(u+1)}{\mathbf{Q}_{\Omega}^{0,1}(u+1) \mathbf{Q}_{\Omega}^{0,0}(u+1)}, \quad E_{\Omega}(u)=\frac{\mathbf{Q}_{\Omega}^{2,2}(u+1) \mathbf{Q}_{\Omega}^{1,1}(u-1)}{\mathbf{Q}_{\Omega}^{2,1}(u+1)} \\
F_{\Omega}(u)=\frac{\mathbf{Q}_{\Omega}^{2,2}(u+1) \mathbf{Q}_{\Omega}^{1,1}(u+1)}{\mathbf{Q}_{\Omega}^{2,1}(u+1)}, \quad \Omega=L, R
\end{gathered}
$$

where we introduced the notation:

$$
\mathbf{Q}_{\Omega}^{k, m}(u)= \begin{cases}\tilde{Q}_{k, m}(u) & \text { in case of } \quad \Omega=R \\ Q^{k, m}(u) & \text { in case of } \quad \Omega=L\end{cases}
$$

Applying the formulae (76) and (79) and putting everything together one obtains series expressions for the T-functions along the line $(1, s)$ :

$T_{2,2}^{2,2}(1, s, u)=\sum_{k=\max (-s, 0)}^{\infty} F_{L}^{(k)}(u+s) F_{R}^{(s+k)}(u-s) Q^{0,0}(u+s+2 k+1) \tilde{Q}_{0,0}(u+s+2 k-1)$.

It can be seen that the T-functions along the line $(1, s)$ can be given only as an infinite series of Q-functions. T-functions for other values of $a$ and $s$ can be determined from (89) by the application of the Bazhanov -Reshetikhin formula (67). As a final result 
we could express the infinitely many T-functions in terms of 9 boundary functions of the hierarchy of Hirota equations of the T-shaped fat-hook of type $(2,2) \odot(2,2)$.

An important remark concerning (89) is in order. In (89) $T_{2,2}^{2,2}(1, s, u)$ is represented by an infinite series, so the convergence of the series has to be dealt with. Obviously for arbitrary boundary functions the series (89) can not be expected to be convergent, but it may be convergent (at least on certain intervals in $u$ ) for certain choices of boundary functions being consistent with QQ-relations (43), (44). Anyway, it can be seen that the usual polynomial spin-chain ansatz for all the boundary functions would lead to divergence. This fact is in accordance with the observation of refs. [24, 28], which states that when the the length of the spin chain in AdS/CFT tends to infinity $T_{a, s \leq 0}(u)$ and $T_{a, s \geq 0}(u)$ cannot be simultaneously finite.

Despite the fact that the formula (89) is difficult to apply in practise, we think that the algorithm for the integration of the Hirota equations described in the previous section together with the TT-, TQ-, and QQ-relations can be used as a tool for the derivation of an NLIE governing the exact finite size spectrum of anomalous dimensions of $\mathcal{N}=4 \mathrm{SYM}$ in the planar limit.

\section{Summary and discussion}

In this paper we dealt with the solution of Hirota equations (2) defined on a Tshaped fat-hook with corner point coordinates $(K, M)$ and $\left(K^{\prime},-M^{\prime}\right)$. The key ingredient to the solution is the existence of a set of auto-Bäcklund transformations for the equations, i.e. transformations which send any solution of the equations to another solution. We introduced four Bäcklund transformations. They send any solution of the Hirota equations defined on the T-shaped fat-hook with corner point coordinates $(K, M)$ and $\left(K^{\prime},-M^{\prime}\right)$ to a solution corresponding to a T-shaped fat hook with different corner point coordinates. (The coordinates $K, M, K^{\prime}, M^{\prime}$ differ.) Specifically, the first Bäcklund transformation (BT1) lowers $K$ by 1 , the second one (BT2) lowers $M$ by 1 , the third one $(\overline{B T 1})$ lowers $K^{\prime}$ by 1 , and finally the fourth one $(\overline{B T 2})$ lowers $M^{\prime}$ by 1 , leaving the other corner point coordinates intact. Thus they define a hierarchy of Hirota equations. Applying the four Bäcklund transformations successively $K+M+K^{\prime}+M^{\prime}$ times, one comes to a collapsed domain being the union of two lines. In this way the original problem gets undressed to a trivial one. In 
case of AdS/CFT, when $K=M=K^{\prime}=M^{\prime}=2$, the different orders, in which one decreases the original T-shaped fat-hook to a trivial domain, correspond to different choices of the basis of simple roots of the symmetry group $S U(2 \mid 2) \otimes S U(2 \mid 2)$.

With the help of the four Bäcklund transformations we derived TT-, TQ-, and QQ-relations for our system and inverting the undressing procedure we could express the infinitely many T-functions of the problem in terms of a few arbitrary functions characterizing the boundary conditions of the hierarchy of Hirota equations (Qfunctions).

In [45] it has been shown that since the Y-functions have infinitely many cuts on the $u$ plane it is natural to think that the Y-system is in fact defined on an infinite genus Riemann surface and the Y-system equations (1) hold only in the strip $-2 g \leq \operatorname{Im} u \leq 2 g$ of the $u$ plant5. In this case the T- and Q-functions have infinitely many branch cuts on the $u$ plane and can also be thought to be defined on infinite genus Riemann surfaces.

The knowledge of the analytic properties of the Y-functions on the infinite genus Riemann surface would allow one to reproduce the TBA equations purely from the $\mathrm{Y}$ system equations. Similarly according to our expectations, the understanding of the analytic properties of the T- and Q-functions on the infinite genus Riemann surface would make it possible to combine the T- and Q-functions of the problem into a new set of functions which have appropriate analytic properties in $u$ to serve as unknown functions of a desired NLIE governing the spectrum of anomalous dimensions in planar $\mathcal{N}=4 \mathrm{SYM}$.

Another application of the results of this paper could be the construction of a lattice model or a spin chain, which probably after some limiting procedure would account for the spectrum of AdS/CFT.

\section{Acknowledgments}

This investigation was supported by the Hungarian National Science Fund OTKA (under T049495).

\footnotetext{
${ }^{5}$ Adapting the notations of 45 to those of this paper
} 


\section{Appendix A}

In this appendix the relations between boundary functions of (24) dictated by "degenerated" cases are listed (see the end of section 3.). The relations are as follows:

$$
\begin{aligned}
\tilde{\mathcal{Q}}_{0}^{k^{\prime}, m^{\prime}}(u) & =Q^{k^{\prime}, m^{\prime}}(u), \quad \mathcal{Q}_{k, m}^{0}(u)=\tilde{Q}_{k, m}(u), \\
\mathcal{Q}_{k, m}^{k}(u) & =\tilde{Q}_{0, m}(u-2 k), \quad \tilde{\mathcal{Q}}_{k}^{k, m}(u)=Q^{0, m}(u-2 k), \\
\tilde{\mathcal{Q}}_{k}^{k^{\prime}, m}(u) & =(-1)^{-m\left(k+k^{\prime}\right)} \mathcal{Q}_{k,-m}^{k^{\prime}}(u+2 m) \quad \tilde{Q}_{0, m}(u)=Q^{0,-m}(u+2 m) .
\end{aligned}
$$

\section{Appendix B}

In this appendix we list additional TQ-type identities between the T-functions and the boundary characterizing functions. These bilinear identities can be derived from the (18) and (21) Bäcklund transformations by taking them at lines lying close to the boundaries.

Taking the second row of (BT1) (18) along the lines $(a, m-1)$ and $(k-1, s)$, one gets the following bilinear relations:

$$
\begin{gathered}
T_{k-1, m}^{k^{\prime}, m^{\prime}}(a, m-1, u) \tilde{\mathcal{Q}}_{k}^{k^{\prime}, m^{\prime}}(u+a+m+1)-(-1)^{m} T_{k, m}^{k^{\prime}, m^{\prime}}(a, m-1, u) \times(91) \\
\tilde{\mathcal{Q}}_{k-1}^{k^{\prime}, m^{\prime}}(u+a+m+1)=T_{k, m}^{k^{\prime}, m^{\prime}}(a+1, m-1, u+1) \tilde{\mathcal{Q}}_{k-1}^{k^{\prime}, m^{\prime}}(u+a+m-1), \quad a \geq k, \\
T_{k, m}^{k^{\prime}, m^{\prime}}(k-1, s+1, u+1) \tilde{\mathcal{Q}}_{k-1}^{k^{\prime}, m^{\prime}}(u+s+k-1)-T_{k, m}^{k^{\prime}, m^{\prime}}(k-1, s, u) \times(92) \\
\tilde{\mathcal{Q}}_{k-1}^{k^{\prime}, m^{\prime}}(u+s+k+1)=T_{k-1, m}^{k^{\prime}, m^{\prime}}(k-2, s+1, u) \tilde{\mathcal{Q}}_{k}^{k^{\prime}, m^{\prime}}(u+s+k+1), \quad s \geq m .
\end{gathered}
$$

Taking the first row of (BT2) (18) at $s=m-1$ and at $a=k-1$, one gets the following TQ-type equations:

$$
\begin{gathered}
T_{k, m}^{k^{\prime}, m^{\prime}}(a, m-1, u+1)(-1)^{m-1} \tilde{Q}_{0, m-1}(u-m-a)-T_{k, m}^{k^{\prime}, m^{\prime}}(a+1, m-1, u) \times \\
\tilde{Q}_{0, m-1}(u-a-m+2)=T_{k, m-1}^{k^{\prime}, m^{\prime}}(a+1, m-2, u+1)(-1)^{a-k} \tilde{Q}_{0, m}(u-a-m), a \geq k, \\
T_{k, m}^{k^{\prime}, m^{\prime}}(k-1, s, u+1) \tilde{Q}_{0, m-1}(u-s-k)-T_{k, m-1}^{k^{\prime}, m^{\prime}}(k-1, s, u+1) \times \\
\tilde{Q}_{0, m}(u-s-k)=T_{k, m}^{k^{\prime}, m^{\prime}}(k-1, s+1, u) \tilde{Q}_{0, m-1}(u-s-k+2), \quad s \geq m .
\end{gathered}
$$


Taking the second row of (21) both for $(\overline{B T 1})$ and $(\overline{B T 2})$ along the line $(a, m)$ for $a \geq k$ one gets the following bilinear relations:

$$
\begin{gathered}
T_{k, m}^{k^{\prime}, m^{\prime}}(a, m-1, u+1) \tilde{\mathcal{Q}}_{k}^{k^{\prime}-1, m^{\prime}}(u+a+m)-T_{k, m}^{k^{\prime}-1, m^{\prime}}(a, m-1, u+1) \times \\
\tilde{\mathcal{Q}}_{k}^{k^{\prime}, m^{\prime}}(u+a+m)=(-1)^{m} T_{k, m}^{k^{\prime}-1, m^{\prime}}(a-1, m-1, u) \tilde{\mathcal{Q}}_{k}^{k^{\prime}, m^{\prime}}(u+a+m+2), a \geq k, \\
T_{k, m}^{k^{\prime}, m^{\prime}-1}(a, m-1, u+1) \tilde{\mathcal{Q}}_{k}^{k^{\prime}, m^{\prime}}(u+a+m)-T_{k, m}^{k^{\prime}, m^{\prime}}(a, m-1, u+1) \times(96), a \geq k . \\
\tilde{\mathcal{Q}}_{k}^{k^{\prime}, m^{\prime}-1}(u+a+m)=(-1)^{m} T_{k, m}^{k^{\prime}, m^{\prime}}(a-1, m-1, u) \tilde{\mathcal{Q}}_{k}^{k^{\prime}, m^{\prime}-1}(u+a+m+2), a
\end{gathered}
$$

Taking the first row of (21) both for $(\overline{B T 1})$ and $(\overline{B T 2})$ at $a=k-1$, one gets the following equations:

$$
\begin{gathered}
T_{k, m}^{k^{\prime}-1, m^{\prime}}(k-1, s, u+1) \tilde{\mathcal{Q}}_{k}^{k^{\prime}, m^{\prime}}(u+s+k)-T_{k, m}^{k^{\prime}, m^{\prime}}(k-1, s, u+1) \times \\
\tilde{\mathcal{Q}}_{k}^{k^{\prime}-1, m^{\prime}}(u+s+k)=T_{k, m}^{k^{\prime}-1, m^{\prime}}(k-1, s-1, u) \tilde{\mathcal{Q}}_{k}^{k^{\prime}, m^{\prime}}(u+s+k+2), \quad s \geq m, \\
T_{k, m}^{k^{\prime}, m^{\prime}}(k-1, s, u+1) \tilde{\mathcal{Q}}_{k}^{k^{\prime}, m^{\prime}-1}(u+s+k)-T_{k, m}^{k^{\prime}, m^{\prime}-1}(k-1, s, u+1) \times \\
\tilde{\mathcal{Q}}_{k}^{k^{\prime}, m^{\prime}}(u+s+k)=T_{k, m}^{k^{\prime}, m^{\prime}}(k-1, s-1, u) \tilde{\mathcal{Q}}_{k}^{k^{\prime}, m^{\prime}-1}(u+s+k+2), \quad s \geq m .
\end{gathered}
$$

Taking the second rows of (18) at $s=-m^{\prime}$ for both the (BT1) and (BT2) cases, the following bilinear equations emerge for $a \geq k^{\prime}$ :

$$
\begin{gathered}
T_{k, m}^{k^{\prime}, m^{\prime}}\left(a,-m^{\prime}+1, u+1\right) \mathcal{Q}_{k-1, m}^{k^{\prime}}\left(u+a+m^{\prime}\right)-T_{k-1, m}^{k^{\prime}, m^{\prime}}\left(a,-m^{\prime}+1, u+1\right) \times \\
\mathcal{Q}_{k, m}^{k^{\prime}}\left(u+a+m^{\prime}\right)=(-1)^{m^{\prime}} T_{k-1, m}^{k^{\prime}, m^{\prime}}\left(a-1,-m^{\prime}+1, u\right) \mathcal{Q}_{k, m}^{k^{\prime}}\left(u+a+m^{\prime}+2\right), \quad a \geq k^{\prime}, \\
T_{k, m-1}^{k^{\prime}, m^{\prime}}\left(a,-m^{\prime}+1, u+1\right) \mathcal{Q}_{k, m}^{k^{\prime}}\left(u+a+m^{\prime}\right)-T_{k, m}^{k^{\prime}, m^{\prime}}\left(a,-m^{\prime}+1, u+1\right) \times \\
\mathcal{Q}_{k, m-1}^{k^{\prime}}\left(u+a+m^{\prime}\right)=(-1)^{m^{\prime}} T_{k, m}^{k^{\prime}, m^{\prime}}\left(a-1,-m^{\prime}+1, u\right) \mathcal{Q}_{k, m-1}^{k^{\prime}}\left(u+a+m^{\prime}+2\right), \quad a \geq k^{\prime} .
\end{gathered}
$$

Taking the first rows of (18) at $a=k^{\prime}-1$ for both the (BT1) and (BT2) cases, one obtains the following TQ-type relations for $s \leq-m^{\prime}$ :

$$
\begin{gathered}
T_{k-1, m}^{k^{\prime}, m^{\prime}}\left(k^{\prime}-1, s, u+1\right) \mathcal{Q}_{k, m}^{k^{\prime}}\left(u-s+k^{\prime}\right)-T_{k, m}^{k^{\prime}, m^{\prime}}\left(k^{\prime}-1, s, u+1\right) \times \\
\mathcal{Q}_{k-1, m}^{k^{\prime}}\left(u-s+k^{\prime}\right)=T_{k-1, m}^{k^{\prime}, m^{\prime}}\left(k^{\prime}-1, s+1, u\right) \mathcal{Q}_{k, m}^{k^{\prime}}\left(u-s+k^{\prime}+2\right), \quad s \leq-m^{\prime}, \\
T_{k, m}^{k^{\prime}, m^{\prime}}\left(k^{\prime}-1, s, u+1\right) \mathcal{Q}_{k, m-1}^{k^{\prime}}\left(u-s+k^{\prime}\right)-T_{k, m-1}^{k^{\prime}, m^{\prime}}\left(k^{\prime}-1, s, u+1\right) \times \\
\mathcal{Q}_{k, m}^{k^{\prime}}\left(u-s+k^{\prime}\right)=T_{k, m}^{k^{\prime}, m^{\prime}}\left(k^{\prime}-1, s+1, u\right) \mathcal{Q}_{k, m-1}^{k^{\prime}}\left(u-s+k^{\prime}+2\right), \quad s \leq-m^{\prime} .
\end{gathered}
$$


Taking the second row of $(\overline{B T 1})(21)$ at $s=-m^{\prime}+1$ and at $a=k^{\prime}-1$, the following equations appear:

$$
\begin{gathered}
T_{k, m}^{k^{\prime}-1, m^{\prime}}\left(a,-m^{\prime}+1, u\right) \mathcal{Q}_{k, m}^{k^{\prime}}\left(u+a+m^{\prime}+1\right)-(-1)^{m^{\prime}} T_{k, m}^{k^{\prime}, m^{\prime}}\left(a,-m^{\prime}+1, u\right) \times \\
\mathcal{Q}_{k, m}^{k^{\prime}-1}\left(u+a+m^{\prime}+1\right)=T_{k, m}^{k^{\prime}, m^{\prime}}\left(a+1,-m^{\prime}+1, u+1\right) \mathcal{Q}_{k, m}^{k^{\prime}-1}\left(u+a+m^{\prime}-1\right), \quad a \geq k^{\prime}, \\
T_{k, m}^{k^{\prime}, m^{\prime}}\left(k^{\prime}-1, s-1, u+1\right) \mathcal{Q}_{k, m}^{k^{\prime}-1}\left(u-s+k^{\prime}-1\right)-T_{k, m}^{k^{\prime}, m^{\prime}}\left(k^{\prime}-1, s, u\right) \times \\
\mathcal{Q}_{k, m}^{k^{\prime}-1}\left(u-s+k^{\prime}+1\right)=T_{k, m}^{k^{\prime}-1, m^{\prime}}\left(k^{\prime}-2, s-1, u\right) \mathcal{Q}_{k, m}^{k^{\prime}}\left(u-s+k^{\prime}+1\right), \quad s \leq-m^{\prime} .
\end{gathered}
$$

Taking the first row of $(\overline{B T 2})(21)$ at $s=-m^{\prime}+1$ and at $a=k^{\prime}-1$, the following bilinear relations emerge:

$$
\begin{gathered}
T_{k, m}^{k^{\prime}, m^{\prime}}\left(k^{\prime}-1, s, u+1\right) Q^{0, m^{\prime}-1}\left(u+s-k^{\prime}\right)-T_{k, m}^{k^{\prime}, m^{\prime}-1}\left(k^{\prime}-1, s, u+1\right) \times \\
Q^{0, m^{\prime}}\left(u+s-k^{\prime}\right)=T_{k, m}^{k^{\prime}, m^{\prime}}\left(k^{\prime}-1, s-1, u\right) Q^{0, m^{\prime}-1}\left(u+s-k^{\prime}+2\right), \quad s \leq-m^{\prime}, \\
T_{k, m}^{k^{\prime}, m^{\prime}-1}\left(a+1,-m^{\prime}+2, u+1\right)(-1)^{a-k^{\prime}} Q^{0, m^{\prime}}\left(u-a-m^{\prime}\right)+T_{k, m}^{k^{\prime}, m^{\prime}}\left(a+1,-m^{\prime}+1, u\right) \times(106) \\
Q^{0, m^{\prime}-1}\left(u-a-m^{\prime}+2\right)=T_{k, m}^{k^{\prime}, m^{\prime}}\left(a,-m^{\prime}+1, u+1\right)(-1)^{m^{\prime}-1} Q^{0, m^{\prime}-1}\left(u-a-m^{\prime}\right), a \geq k^{\prime} .
\end{gathered}
$$

\section{References}

[1] J. M. Maldacena, "The large N limit of superconformal field theories and supergravity," Adv. Theor. Math. Phys. 2 (1998) 231 [Int. J. Theor. Phys. 38 (1999) 1113], hep-th/9711200; S. S. Gubser, I. R. Klebanov and A. M. Polyakov, "Gauge theory correlators from non-critical string theory," Phys. Lett. B 428 (1998) 105, hep-th/9802109 ; E. Witten, "Anti-de Sitter space and holography," Adv. Theor. Math. Phys. 2 (1998) 253, hep-th/9802150.

[2] J. A. Minahan and K. Zarembo, "The Bethe-ansatz for $\mathrm{N}=4$ super YangMills," JHEP 0303, 013 (2003), hep-th/0212208.

[3] N. Beisert, C. Kristjansen and M. Staudacher, "The dilatation operator of N = 4 super Yang-Mills theory," Nucl. Phys. B 664 (2003) 131, [hepth/ 0303060].

[4] A. V. Belitsky, S. E. Derkachov, G. P. Korchemsky and A. N. Manashov, "Quantum integrability in (super) Yang-Mills theory on the light-cone," Phys. Lett. B 594 (2004) 385 hep-th/0403085. 
[5] I. Bena, J. Polchinski and R. Roiban, "Hidden symmetries of the $A d S_{5} \times S^{5}$ superstring," Phys. Rev. D 69 (2004) 046002, [hep-th/0305116].

[6] L. Dolan, C. R. Nappi and E.Witten, "A relation between approaches to integrability in superconformal Yang-Mills theory," JHEP 0310 (2003) 017, hep-th/0308089].

[7] N. Beisert and M. Staudacher, "The N = 4 SYM integrable super spin chain," Nucl. Phys. B 670 (2003) 439 arXiv:hep-th/0307042.

[8] V. A. Kazakov, A. Marshakov, J. A. Minahan and K. Zarembo, "Classical/quantum integrability in AdS/CFT," JHEP 0405, 024 (2004) arXiv:hep-th/0402207].

[9] G. Arutyunov, S. Frolov and M. Staudacher, "Bethe Ansatz for Quantum Strings," JHEP 0410:016 (2004), arXiv:hep-th/0406256.

[10] N. Beisert, V. A. Kazakov, K. Sakai and K. Zarembo, "The algebraic curve of classical superstrings on $A d S_{5} \times S^{5}$," Commun. Math. Phys. 263, 659 (2006) arXiv:hep-th/0502226.

[11] N. Beisert, V. A. Kazakov, K. Sakai and K. Zarembo, "Complete spectrum of long operators in N = 4 SYM at one loop," JHEP 0507, 030 (2005) arXiv:hep-th/0503200.

[12] N. Beisert, V. Dippel and M. Staudacher, "A novel long range spin chain and planar N = 4 super Yang-Mills," JHEP 0407, 075 (2004) hep-th/0405001.

[13] M. Staudacher, "The factorized S-matrix of CFT/AdS," JHEP 0505, 054 (2005) hep-th/0412188.

[14] N. Beisert and M. Staudacher, "Long-range $P S U(2,2 \mid 4)$ Bethe ansatz for gauge theory and strings," Nucl. Phys. B 727, 1 (2005) [hep-th/ 0504190].

[15] M. Luscher, "Volume Dependence of the Energy Spectrum in Massive Quantum Field Theories. 2. Scattering States," Commun. Math. Phys. 105, 153 (1986). 
[16] M. Luscher, "Volume Dependence Of The Energy Spectrum In Massive Quantum Field Theories. 1. Stable Particle States," Commun. Math. Phys. 104, 177 (1986).

[17] J. Ambjorn, R. A. Janik and C. Kristjansen, "Wrapping interactions and a new source of corrections to the spin-chain / string duality," Nucl. Phys. B 736, 288 (2006) arXiv:hep-th/0510171.

[18] R. A. Janik and T. Lukowski, "Wrapping interactions at strong coupling " the giant magnon," Phys. Rev. D 76, 126008 (2007) [arXiv:0708.2208 [hep-th]].

[19] Y. Hatsuda and R. Suzuki, "Finite-Size Effects for Dyonic Giant Magnons," Nucl. Phys. B 800, 349 (2008) [arXiv:0801.0747 [hep-th]].

[20] N. Gromov, S. Schafer-Nameki and P. Vieira, "QuantumWrapped Giant Magnon," Phys. Rev. D78, 026006 (2008) [arXiv:0801.3671 [hep-th]].

[21] M. P. Heller, R. A. Janik and T. Lukowski, "A new derivation of Luscher F-term and fluctuations around the giant magnon," JHEP 0806, 036 (2008) arXiv:0801.4463 [hep-th]].

[22] G. Arutyunov and S. Frolov, "On String S-matrix, Bound States and TBA", JHEP0712:024 (2007), arXiv:0710.1568 [hep-th]]

[23] G. Arutyunov and S. Frolov, "String hypothesis for the $A d S_{5} x S^{5}$ mirror," JHEP 0903:152 (2009), arXiv:0901.1417 [hep-th]].

[24] N. Gromov, V. Kazakov and P. Vieira, "Integrability for the Full Spectrum of Planar AdS/CFT", arXiv:0901.3753 [hep-th]] accepted for publication in Phys. Rev. Lett.

[25] N. Gromov, V. Kazakov A. Kozak and P. Vieira, "Integrability for the Full Spectrum of Planar AdS/CFT II", arXiv:0902.4458 [hep-th]]

[26] G. Arutyunov and S. Frolov, "Thermodynamic Bethe Ansatz for the $A d S_{5} \times S^{5}$ Mirror Model", JHEP 0905:068 (2009) [arXiv:0903.0141 [hep-th]] 
[27] D. Bombardelli, D. Fioravanti and R. Tateo, "Thermodynamic Bethe Ansatz for planar AdS/CFT: a proposal", arXiv:0902.3930v2 [hep-th]]

[28] N. Gromov, V. Kazakov and P. Vieira, "Finite Volume Spectrum of 2D Field Theories from Hirota Dynamics ", arXiv:0812.5091 [hep-th]]

[29] A. Klümper and P. Pearce, "Conformal weights of RSOS lattice models and their fusion hierarchies," Physica A 183 (1992) 304-350;

A. Kuniba, T. Nakanishi and J. Suzuki, "Functional relations in solvable lattice models I: Functional relations and representation thoery," Int. J. Mod. Phys. A9 5215-5312 (1994) arXiv:hep-th/9309137.

[30] Z. Tsuboui, "Analytic Bethe Ansatz and functional equations for Lie superalgebra $s l(r+1 \mid s+1)$," J. Phys A:Math. Gen. 30 (1997) 7975-7991; "Analytic Bethe Ansatz and functional equations associated with any simple root systems of the Lie superalgebra $s l(r+1 \mid s+1)$," Physica A 252 (1998) 565-585.

[31] F. Gohmann, A. Seel, "A note on the Bethe Ansatz solution of the supersymmetric t-J model," Czech. J. Phys. 53 (2003) 1041-1046, arXiv:cond-mat/0309138

[32] R. Hirota, "Discrete analogue of a generalized Toda equation", Journ. of the Phys. Soc. of Japan, 50 (1981), 3785-3791.

[33] T. Miwa, "On the Hirota's difference equations", Proc. Japan Acad. Ser. A58 (1982) 9-12.

[34] E. Date, M. Jimbo and T. Miwa, "Method for generating discrete soliton equations I", J. Phys. Soc. Japan 51 (1982) 4116-4127.

[35] M. Jimbo and T. Miwa, "Solitons and infinite dimensional Lie algebras", Publ. RIMS 19 (1983) 943-1001.

[36] S. Saito and N. Saitoh, "Lineraization of bilinear difference equations", Phys. Lett. A120 (1987) 322-326; "Gauge and dual symmetries and linearization of Hirota's bilinear equations", J. Math. Phys. 28 (1987) 1052-1055. 
[37] A. Zabrodin, "Discrete Hirota's equation in integrable models", Int. J. Mod. Phys. B 11 (1997) 3125-3158, [arXiv.org:hep-th/9610039]; " Hirota equation and Bethe ansatz", Theor. Math. Phys. 116 (1998) 782-819.

[38] I. Krichever, O.Lipan, P.Wiegmann, A. Zabrodin, "Quantum Integrable Systems and Elliptic Solutions of Classical Discrete Nonlinear Equations ", Commun.Math.Phys. 188 (1997) 267-304, [ arXiv:hep-th/9604080]

[39] V. Kazakov, A. Sorin and A. Zabrodin, "Supersymmetric Bethe Ansatz and Baxter Equations from Discrete Hirota Dynamics", Nucl.Phys.B790 345-413 (2008), arXiv:hep-th/0703147

[40] Z. Bajnok and R. A. Janik, "Four-loop perturbative Konishi from strings and finite size effects for multiparticle states", Nucl.Phys. B807 625-650 (2009), arXiv:0807.0399 [hep-th]]

[41] Z. Bajnok, R. A. Janik and T. Lukowski, "Four loop twist two, BFKL, wrapping and strings", Nucl.Phys.B816 376-398 (2009), [arXiv:0811.4448v4 [hep-th]]

[42] M. Beccaria, V. Forini, T. Lukowski, S. Zieme, "Twist-three at five loops, Bethe Ansatz and wrapping", JHEP 0903:129 (2009), arXiv:0901.4864v2 [hep-th]]

[43] V. Bazhanov and N. Reshetikhin, "Restricted solid-on-solid models connected with simply laced algebras and conformal field theory", J. Phys. A: Math. Gen. 23 (1990), 1477-1492.

[44] A. Kirillov and N. Reshetikhin, J. Phys. A20, 1565 (1987),

V. Bazhanov and N. Reshetikhin, Int. J. Mod. Phys. A4, 115 (1989),

R. J. Baxter, Exactly solved models in statistical mechanics (Academic Press, 1982).

[45] S. Frolov and R. Suzuki, "Temperature quantization from the TBA equations" arXiv:0906.0499 [hep-th]] 